\title{
Activités de formation des enseignants novices et trajectoire de satisfaction professionnelle : conceptualisation et illustration
}

Activities in vocational training and the satisfaction trajectory of novice teachers: theoretical conceptualization and empirical illustration

Jérôme Amathieu et Sébastien Chaliès

\section{(2) OpenEdition}

\section{Journals}

Édition électronique

URL : https://journals.openedition.org/activites/6670

DOI : 10.4000/activites.6670

ISSN : 1765-2723

Éditeur

ARPACT - Association Recherches et Pratiques sur les ACTivités

Référence électronique

Jérôme Amathieu et Sébastien Chaliès, «Activités de formation des enseignants novices et trajectoire de satisfaction professionnelle : conceptualisation et illustration », Activités [En ligne], 18-2 | 2021, mis en ligne le 15 octobre 2021, consulté le 13 septembre 2022. URL : http://journals.openedition.org/ activites/6670 ; DOI : https://doi.org/10.4000/activites.6670

Ce document a été généré automatiquement le 13 septembre 2022.

Creative Commons - Attribution - Pas d'Utilisation Commerciale - Pas de Modification 4.0 International - CC BY-NC-ND 4.0

https://creativecommons.org/licenses/by-nc-nd/4.0/ 


\section{Activités de formation des enseignants novices et trajectoire de satisfaction professionnelle : conceptualisation et illustration}

Activities in vocational training and the satisfaction trajectory of novice teachers: theoretical conceptualization and empirical illustration

Jérôme Amathieu et Sébastien Chaliès

NOTE DE L'ÉDITEUR

Article soumis le 11/02/2021, accepté le 28/06/2021

\section{Introduction}

1 La multiplication de rapports relatifs au bien-être des travailleurs en France établit une relation entre la santé des enseignants et la nature de leur activité menée en situation de travail.

2 Différents rapports institutionnels proposent une vision du travail comme contributeur de la santé. Ces approches établissent des liens étroits entre la santé des enseignants et la nature de leurs activités en situation de travail.

3 La qualité de vie au travail occupe désormais une place grandissante dans la compréhension du malaise ou bien être au travail (Lanouzière, 2019). Après la prédominance d'approches privilégiant les risques dans les études du malaise et du bien-être des travailleurs en France marqué notamment par le rapport Nasse-Légeron 
sur la «détermination, la mesure et le suivi des risques psychosociaux au travail» (2008), les approches privilégiées actuellement mettent au cœur de la réflexion la qualité de vie au travail. Ces approches privilégient une vision développementale du travail comme contributeur de santé et non comme potentiellement porteur de risques à éviter ou limiter (Lanouzière, 2019). C'est ici que trouve sens notre définition de la santé, labile (Canguilhem, 1943) et conquise en quelque sorte par le professionnel de par son engagement dans un processus de normativité (Le Blanc, 2008). Le rapport Lachman (2010) intitulé "Bien-être et efficacité au travail» est à ce titre particulièrement significatif. Dans ces nouvelles approches, l'ambition est de travailler à accroitre la capacité des travailleurs à agir sur leurs conditions de travail afin d'accroitre leur qualité de vie professionnelle (Coutrot, 2018). Dans cette optique, le travail favoriserait le développement des capacités et le bien-être des salariés. Une qualité de travail perçue plus importante contribuerait ainsi à la satisfaction [par exemple Billaudeau \& Vercambre-Jacquot (2015) dans le domaine de l'enseignement]. Une lecture attentive des productions scientifiques étudiant les relations entre santé et travail souligne des liens étroits entre santé et satisfaction professionnelle des travailleurs (notamment les travaux de la clinique de l'activité : Clot, \& Lhuilier, 2015 ; Dejours, 2016 ; Lhuillier, \& Litim, 2009).

4 Au cœur de cette préoccupation, nous travaillerons indirectement à interroger les liens santé-travail en étudiant les fluctuations de la satisfaction d'enseignants novices (EN) engagés dans un dispositif d'accompagnement tutoral singulier ${ }^{1}$. Introduite notamment par les travaux de Locke (1976), la satisfaction professionnelle se définit généralement comme un "état positif ou agréable résultant de l'appréciation par une personne de son travail ou de ses expériences » (p. 130). Elle nait en effet d'un plaisir ou d'une gratification suite à la perception par le travailleur du caractère adéquat de son travail (Lee, Lee, Liao, \& Chang, 2009; Yennek, 2015). Si la satisfaction professionnelle repose sur une diversité de facteurs (Duffy, \& Lent, 2009), la dimension relative à la nature du travail engagé semble avoir une influence sur l'engagement du formé dans sa formation.

Dans le domaine de l'enseignement, de multiples travaux (pour une synthèse voir: Amathieu, \& Chaliès, 2014) ont montré que l'accroissement de la satisfaction professionnelle engendre par exemple une réduction du stress (Boyle, Borg, Falzon, \& Baglioni, 1995) ou encore une élévation du sentiment d'efficacité personnelle (Jones Johnson, \& Johnson, 2000). À l'inverse, certains travaux ont montré qu'un niveau élevé d'insatisfaction professionnelle pouvait être source d'épuisement et de stress chez les enseignants (Skaalvik, \& Skaalvik, 2015). Parallèlement, des travaux se sont attachés à étudier les facteurs influençant la satisfaction professionnelle des enseignants (Duffy, \& Lent, 2009). Parmi ces facteurs, les actions de formation qui leur sont proposées occupent de plus en plus une place de choix dans la littérature (Gaziel, \& WassersteinWarnet, 2005). Le postulat est fait que, mieux formés, les enseignants s'engagent dans un travail de plus grande qualité et, en retour malgré les efforts entrepris, s'en trouvent davantage satisfaits. L'étude menée par Nir et Bogler (2008) est, à ce titre, tout à fait significative. Dans cette étude quantitative menée auprès de 841 enseignants en Israël, les auteurs montrent les retombées de la mise en œuvre d'un dispositif de formation singulier (dispositif engagé sur trois années consécutives, centré sur les préoccupations professionnelles des enseignants et mis en œuvre au sein même de leurs établissements scolaires) sur leur satisfaction professionnelle. Une analyse détaillée de cette étude permet néanmoins de soulever deux réserves. Ces réserves sont d'autant plus significatives qu'elles peuvent adresser à l'ensemble des études réalisées 
dans le champ. D'une part, une réserve méthodologique peut être mise en avant: l'évaluation du niveau de satisfaction des enseignants est en effet effectuée quantitativement à partir de réponses à des questionnaires standardisés (voir par exemple: Lent, \& Brown, 2008) se voulant couvrir l'ensemble des expériences professionnelles du travailleur. Elle ne permet donc pas de réellement rendre compte du caractère effectif de la transformation de l'activité professionnelle des enseignants en contexte de classe (sauf ponctuellement), transformation source potentiellement de satisfaction. D'autre part, une réserve théorique peut être mise en exergue. Comme dans la plupart des travaux du domaine, la satisfaction professionnelle est assimilée à un état psychologique stable et par-là même fondamentalement "a situé ». Autrement dit, le postulat selon lequel une fois «acquise " par les travailleurs, la satisfaction ne serait plus soumise aux circonstances de réalisation du travail, pourtant sans cesse renouvelées, n'est sans doute pas assez discutée.

6 L'objet premier de cet article est d'essayer de contribuer au dépassement de ces deux réserves. Pour ce faire, cet article propose successivement (i) une conceptualisation théorique originale permettant d'appréhender selon un point de vue qualitatif le couplage activité(s) - satisfaction, (ii) une démarche méthodologique permettant de rendre compte du caractère situé et dynamique de ce couplage et, enfin, (iii) une illustration et une discussion du caractère heuristique des avancées théoriques et méthodologiques à partir de la présentation de résultats empiriques extraits d'une étude de cas.

\section{Cadre théorique}

7 Le cadre théorique adopté emprunte ses principaux postulats à un programme de recherche mené en anthropologie culturaliste (pour plus de détails: Chaliès \& Amathieu, 2013; Chaliès \& Bertone, 2017). Ce programme a déjà donné lieu à un nombre significatif de travaux dans le champ de la formation professionnelle des adultes, en particulier des enseignants. Compte tenu de l'objet de l'article, quatre de ces postulats sont ci-après explicités.

\subsection{Premier postulat : travailler et se former nécessitent de s'engager dans un double régime de réflexivité}

$8 \mathrm{Au}$ sein de ce cadre théorique, travailler et/ou se former au travail revient à mener des actions " gouvernées par des règles » et/ou à réaliser des actions qui sont «le suivi de règles» (Ogien, 2007). Ces règles sont des "expériences normatives situées» (Lähteenmäki, 2003) acceptées par la communauté professionnelle, rendant intelligibles, anticipables et évaluables les actions de chacun (Livet, 1993). Les règles constituent plus précisément la syntaxe de ces actions considérées comme efficaces par la communauté professionnelle considérée. Elles font autorité pour chaque travailleur, car elles constituent autant de standards de correction au sein de la communauté. Elles ne déterminent cependant pas leur propre application. Chaque acteur peut en effet à tout instant en rejeter le suivi (Descombes, 2004). Pour chaque acteur en formation, ces règles constituent une sorte de "grammaire » expérientielle, non encore maîtrisée, qui sert de véritable mètre-étalon pour pouvoir reconnaître et/ou juger de la conformité des actions entreprises aux prescriptions de métier (Clot, 2008). Deux modalités 
d'institution des règles peuvent être ici succinctement délimitées. En premier lieu, une institution que l'on pourrait considérée d'« explicite " existe. Les règles sont ainsi formalisées à l'occasion de discussions et/ou d'échanges plus ou moins informels entre professionnels. Une fois instituées elles peuvent d'ailleurs devenir objet des situations de formation comme celle par exemple de tutorat. Dans certains travaux relevant de la clinique de l'activité (par exemple: Clot \& Scheller, 2000), cette possibilité d'élaboration des règles est pour ainsi dire provoquée lors de la réalisation d'entretiens d'autoconfrontation croisés afin d'élaborer le «genre professionnel » en confrontant les «styles personnels ». Parallèlement, une institution des règles se réalise de façon implicite par un alignement informel des pratiques entre professionnels (Chauvité, 2004).

Cette double possibilité d'institution des règles tient en réalité de la possibilité pour l'acteur de s'engager dans un double registre de réflexivité par rapport à celles-ci. Dans certaines situations professionnelles, il est engagé dans un régime de réflexivité où il réalise des actions qui sont "gouvernées par les règles» (Ogien, 2007). Ce faisant, il signifie les faits et occurrences de son contexte de travail de façon immédiate et préréflexive (Legrand, 2005). Selon Taylor (1997), l'acteur parvient à une " compréhension non formulée » du monde dans lequel il agit qui (i) «permet de trouver un sens aux choses et aux actions (...) entièrement informulé » (p. 560) et (ii) «peut servir de base à une formulation nouvelle " (p.564), le cas échéant. Dans un grand nombre de situations de travail, l'acteur réalise des actions dont l'apprentissage s'est fait implicitement (Lave, \& Wenger, 1991) par l'intermédiaire d'interactions non verbales et/ou d'alignements informels avec la pratique d'autres travailleurs (Rogoff, Matusov, \& White, 1996). Ce régime de réflexivité est de l'ordre de "l'inhérence», ou de l'immanence des règles à l'action et à la signification, ce qui n'enlève nullement la possibilité de l'acteur d'énoncer ces règles, pour peu qu'on l'incite à le faire dans des conditions d'échanges favorables (Bertone \& Chaliès, 2015).

Dans d'autres situations professionnelles, l'acteur est engagé dans un régime de réflexivité qui lui permet de se représenter et de rendre compte des raisons de son action et donc des règles qu'il suit lorsqu'il agit (Wittgenstein, 1992). Ce faisant il signifie les faits et occurrences de son contexte professionnel de façon consciente et narrative. Ce régime de réflexivité est de l'ordre de "l'appréhension de l'inhérence » ou de la transcendance des règles à l'action, ce qui ne nécessite pas que l'acteur possède la capacité pratique à réaliser l'action gouvernée par les règles correspondantes. La correspondance entre les énoncés des règles de l'action et la réalisation des actions réglées est effective lorsque l'acteur a appris non seulement à "suivre des règles " (Ogien, 2007) par l'intermédiaire d'un enseignement, accompagné de réélaborations discursives et réflexives, mais aussi à produire les actions considérées comme conformes aux règles et qui satisfont la communauté professionnelle de référence.

\subsection{Deuxième postulat : toute formation professionnelle par alternance repose sur des activités consubstantielles d'enseignement - apprentissage de règles}

11 La possibilité d'un engagement des formés dans une pratique réflexive à propos de leur travail nécessite un apprentissage préalable de règles leur permettant de signifier correctement les expériences qu'ils ont pu vivre. Cet apprentissage nécessite 
théoriquement l'engagement des formateurs dans une activité "d'enseignement ostensif » (Wittgenstein, 2004) par laquelle ils fondent la signification d'expériences considérées comme exemplaires, qui sont énoncées par des règles. Pour ce faire, les formateurs dressent pour chacune de ces expériences un «lien de signification " (Bertone, Chaliès, \& Clot, 2009) liant précisément, (i) cette expérience langagière permettant de nommer un fait ou d'énoncer une action, (ii) les exemples décrits, donnés à observer ou imiter, accompagnés des déictiques permettant de les désigner et identifier («là », « maintenant », etc.), qui sont utilisés comme des échantillons ou des exemples emblématiques des règles et (iii) les résultats des actions réalisées, usuellement associés ou attendus dans la communauté des enseignants expérimentés. Ces liens de significations enseignés (liens entre expériences permettant de nommer exemplariser - fixer les résultats attendus) par les formateurs peuvent ensuite se transformer en véritables expériences "étalon» (Williams, 2002) lorsque les formés sont au travail. Ils peuvent alors s'appuyer sur ces échantillons d'expériences exemplaires pour signifier et juger les événements observés et s'y adapter comme il est attendu de le faire dans la communauté professionnelle considérée.

Les formateurs ne peuvent toutefois se satisfaire de cette activité d'enseignement. Ils doivent ensuite s'engager dans une nouvelle activité « d'accompagnement des premiers suivis " des règles par les formés. Cet accompagnement se réalise post-actu, lors de situations de formation et/ou, lors du retour des formés au travail. Par cet accompagnement, les formateurs visent à rendre possible la réalisation des actions attendues par les formés (par exemple en aménageant la situation de travail), mais aussi et surtout à faire en sorte que les premiers suivis des règles de métier aboutissent aux résultats attendus y étant usuellement associés. Ce n'est qu'au travers du constat de ces résultats que les formés pourront alors rattacher une intention professionnelle concrète aux règles préalablement enseignées par les formateurs et suivies par eux (Cash, 2009). Lors de ces premiers suivis, les formateurs en « contrôlent » la conformité au regard des règles enseignées et s'engagent, si nécessaire, dans une activité d'« explication ostensive » (Davis, 2009). Ils s'efforcent alors de multiplier les exemples décrits de sorte à lever les éventuelles mésinterprétations. C'est seulement à ce moment de l'apprentissage des règles de métier que les formés sont en mesure de s'émanciper de leur contrôle. Ils parviennent alors à construire un système d'interprétations des règles (Winch, 2009) qui autorise un usage « extensif » des liens de signification construits et appris au-delà des situations d'apprentissage originelles. C'est sur la base de son identification d'un « air de famille » entre les circonstances de la situation en cours et celles constitutives des situations originelles de formation que cet usage extensif est possible. À partir des liens de signification appris, les formés ordonnent alors finalement un réseau complexe de ressemblances, « comprennent » la nouvelle situation en cours, et, in fine, parviennent à produire des activités conformes aux attentes de la communauté professionnelle, tout en s'émancipant progressivement de leur contrôle. Lors de ces situations de développement, les formateurs cherchent alors à aider les formés à s'engager dans des suivis singuliers des règles apprises et à se jouer de leur signification originelle pour mieux en user dans des circonstances de travail sans cesse renouvelées et inattendues. 


\subsection{Troisième postulat : la formation accroît la satisfaction professionnelle en permettant aux formés d'obtenir par leur activité les résultats attendus par la communauté}

13 La satisfaction est à considérer comme l'expression d'une appréciation. En effet, «l'appréciation esthétique ne consiste en rien d'autre pour Wittgenstein qu'en l'expression de cette satisfaction; le véritable jugement esthétique est celui qui évalue la correction ou la justesse d'une exécution artistique»(Chauviré, 1992, p. 66). Autrement dit, lorsqu'il porte un jugement positif sur son activité professionnelle, le travailleur donne accès à sa satisfaction, résultat d'une activité préalable d'appréciation de ce qu'il a fait. Sa satisfaction peut être considérée comme résultant d'une activité d'appréciation qui compare, d'une part, les résultats obtenus suite à l'activité engagée et, d'autre part, les résultats attendus que lui avaient initialement enseignés les formateurs. Dans le cas de cette authentification rétrospective, le travailleur éprouve donc de la satisfaction (Chauviré, 2010). Dans le cadre des différentes étapes préalablement définies en matière d'activités des formateurs, la satisfaction peut être doublement située. Elle peut, tout d'abord, advenir lorsque le travailleur, engagé dans l'apprentissage d'une nouvelle règle, constate suite aux suivis de celle-ci les résultats attendus que les formateurs lui avaient initialement enseignés. Elle peut, par ailleurs, advenir lors de situations de formation concourant à l'émergence de nouveaux usages de règles non plus en cours d'apprentissage, mais suite à celui-ci c'est-à-dire lors du développement professionnel.

14 En élargissant les circonstances dans lesquelles il est possible de "faire jouer » les règles, le travailleur parvient à les ajuster tout en aboutissant aux résultats attendus au sein de la communauté professionnelle. Cet usage «extensif» des règles apprises est aussi théoriquement source de satisfaction. C'est en effet au cours de cet usage «extensif» des règles apprises que le travailleur se trouve consubstantiellement satisfait de son activité. Une première forme d'insatisfaction à propos du travail réalisé émerge lorsque ces usages renouvelés des règles ne parviennent pas à obtenir les résultats obtenus dans les circonstances antérieures et actuellement attendus. C'est dans ces cas ordinaires de formation qu'un « problème » émerge (Olszewska, \& Quéré, 2009), accompagné d'un jugement d'insatisfaction des actions réalisées. Une deuxième forme d'insatisfaction peut être engendrée par l'organisation elle-même du travail en formation (contraintes d'efficacité factices imposées sur la base de référentiels d'activité imposés et non liés aux règles du métier, prescription de qualité trahissant la qualité attendue du travail au profit d'un plus grand rendement ou d'une rationalisation du travail, etc.). Cette insatisfaction profonde et durable produit des " empêchements ( (Clot, 2008) ou des dilemmes chez le travailleur, qui se trouve ainsi dans l'impossibilité de suivre correctement les règles apprises et en souffre (Donahue, 1999). Bien que les cas "d'empêchement" des travailleurs soient récurrents et cruciaux pour la compréhension des phénomènes de souffrance; nous nous limiterons à l'étude de cas « d'insatisfaction » que l'on retrouve dans des circonstances ordinaires de formation dans lesquelles des "problèmes » d'efficacité se posent, sans que la santé ne soit affectée profondément et durablement. 


\subsection{Quatrième postulat : Chaque couplage activité - satisfaction ne peut être appréhendé que comme partie prenante d'une trajectoire}

La satisfaction du travailleur peut être théoriquement considérée comme tout à la fois, dynamique et située, car nécessairement consubstantielle aux résultats de son activité. Pour rendre compte de ce double caractère, la notion de "trajectoire " peut être empruntée à la sociologie du travail et plus particulièrement aux propositions faites par Strauss (1992). Selon cet auteur, il est en effet possible de « différencier le cours d'une maladie (son évolution, ses étapes et ses symptômes) de la trajectoire de la maladie. Il identifie sous le terme de trajectoire de la maladie aussi bien le développement physiologique de cette dernière (cours d'une maladie), que l'organisation du travail déployée à suivre ce cours et le retentissement du travail et de son organisation sur ceux qui y sont impliqués» (p. 143). En ce sens, «la trajectoire ordonne l'ensemble des évènements qui interviennent pour affronter la maladie. Elle implique une gestion, et donc un « travail complexe de trajectoire» (p. 187).

Sur cette base, il est donc possible de considérer que la trajectoire de satisfaction dépasse la stricte succession d'états de satisfaction ou d'insatisfaction pour recouvrir aussi le "travail de trajectoire ", c'est-à-dire les activités menées par les différents acteurs y contribuant tout au long de son déroulement. Autrement dit, la trajectoire de satisfaction intègre tout à la fois et à chaque instant un niveau de (d') (in)satisfaction et la (ou les) activité(s) située(s) des acteurs (formés et/ou formateurs) y ayant contribué. Plus en détail, chaque trajectoire de satisfaction peut être appréhendée comme la succession de différentes « phases » (Strauss, 1992) ou « épisodes » (Fillietaz, 2009), lors desquelles le travail de trajectoire des acteurs est mené. Ces différentes phases sont introduites et clôturées par des " points de trajectoire » (Strauss, Fagerhaugh, Suczek, \& Wiener, 1985), c'est-à-dire des niveaux temporaires de satisfaction.

\section{Méthode}

Afin d'illustrer, d'une part, le caractère heuristique des postulats théoriques détaillés en amont pour appréhender selon un point de vue qualitatif le couplage activité(s) satisfaction et, d'autre part, la démarche méthodologique y étant associée pour rendre compte du caractère situé et dynamique de ce couplage, nous nous appuyons sur la présentation de résultats extraits d'une étude de cas. Les principales caractéristiques de cette étude sont les suivantes.

\subsection{Dispositif expérimental}

Le dispositif support à l'étude était celui dit du « tutorat mixte » mis en place au sein de chaque Institut National Supérieur du Professorat et de l'Éducation (INSPE) lors de l'année de titularisation des enseignants novices EN ( $2^{\text {nde }}$ année de Master « Métier de l'Enseignement, de l'Éducation et de la Formation »). Ce dispositif avait été sélectionné, car il était le seul à promouvoir l'institution de collectifs de formateurs (une dyade constituée d'un formateur universitaire (FU) et d'un tuteur de terrain (T)) pour accompagner chaque EN simultanément ou séparément à l'université et/ou en établissement scolaire. Ce dispositif existant a été transformé dans le cadre de cette étude. Les principaux aménagements concernent le nombre, la nature et la fréquence 
des visites. Les visites de formation menées en établissement scolaire par la dyade de formateurs ont été plus particulièrement aménagées. Chaque dyade était invitée à réaliser quatre visites massées dans le temps de sorte à intensifier le suivi des EN. Ces visites à caractère formatif étaient structurées autour de deux temps successifs : la leçon menée par l'EN sous l'observation du $\mathrm{T}$ et duFU et l'entretien de conseil pédagogique (ECP) y faisant suite. Entre ces visites, le T et le FU menaient séparément, respectivement en établissement scolaire et à l'ESPE, un travail de formation avec l'EN.

Figure 1 : Dispositif de tutorat mixte mis en place.

Figure 1: Mixed tutoring system

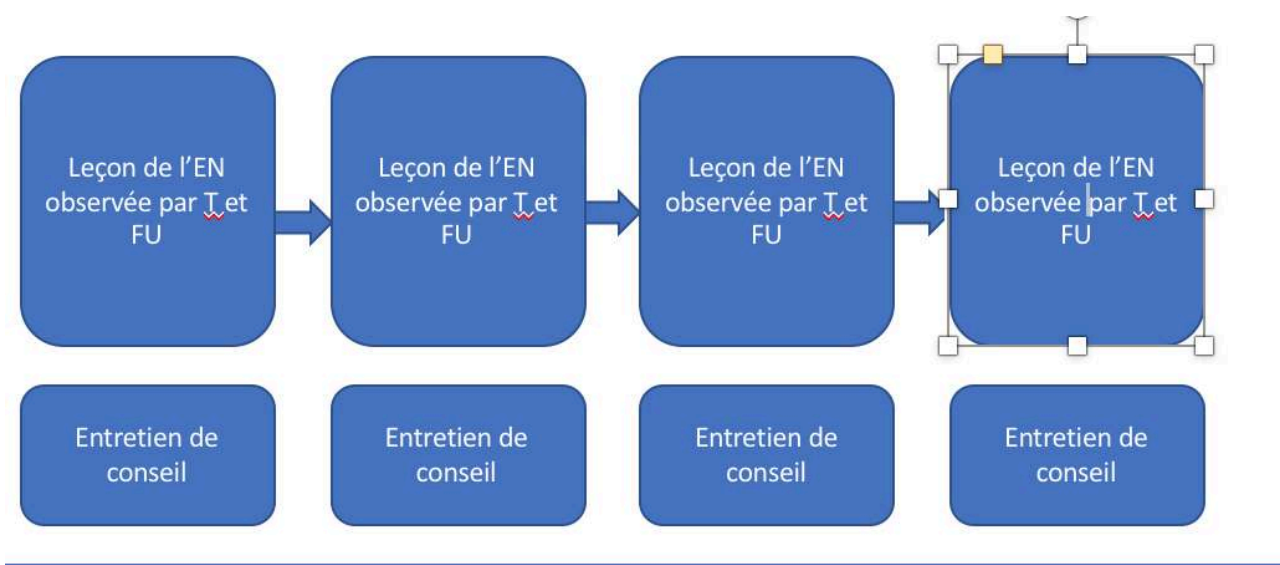

\section{Travail de formation du FU à l'université}

Travail de formation du T en établissement

\subsection{Participants}

19 L'étude de cas exploitée avait été réalisée avec deux triades volontaires constituées d'une EN, d'une T et d'un FU. Âgées de 25 ans, les EN avaient pour la première fois la responsabilité de leurs propres classes, pendant une année scolaire complète. Les deux $\mathrm{T}$ étaient considérées comme des enseignantes expérimentées (respectivement: 16 et 22 années d'expérience), mais aussi comme des $\mathrm{T}$ expérimentées. Les deux FU étaient des formateurs de l'ESPE. Leur expérience de formateur était respectivement de 13 et 20 ans. Ils avaient, tous les deux, une expérience d'enseignant (respectivement 14 et 18 années) et de $\mathrm{T}$ (respectivement 4 et 6 années).

\subsection{Recueil de données}

Comme pour l'ensemble des études menées au sein du programme de recherche, une méthode singulière de recueil et de traitement des données a été adoptée (pour plus de détails: Bertone \& Chaliès, 2015). Deux types de données ont été recueillis puis retranscrits verbatim afin d'être traités. Pour chacune des quatre visites effectuées au sein du dispositif expérimental, la leçon et l'ECP ont été filmés à l'aide d'une caméra vidéo et d'un micro HF. Sur la base de ces données extrinsèques, des données intrinsèques ont ensuite été recueillies lors d'entretiens d'autoconfrontation (EAC) de 
chacun des acteurs à l'enregistrement audio vidéo des leçons et/ou des ECP. Pour chaque visite de formation, chacun des acteurs de la triade a ainsi réalisé un EAC relatif à l'ECP. Seuls les EN ont mené un EAC relatif à la leçon. Au final, 16 EAC, d'une durée de 45 'à une heure, ont été menés pour chaque triade.

Dans le détail, chaque EAC a été conduit de façon à pouvoir reconstituer a posteriori les règles suivies par les acteurs en cours d'ECP ou de leçon. Par un questionnement semistructuré, le chercheur a incité plus précisément l'acteur interviewé à tout d'abord signifier les actions réalisées ou observées (par exemple : «Que fais-tu là ? »). Il l'a incité ensuite à porter un jugement de satisfaction (c'est satisfaisant ou insatisfaisant) sur les actions préalablement signifiées. En lui demandant des précisions (par exemple : «Je ne comprends pas pourquoi tu considères cette action intéressante, peux-tu reprendre?»), ou en jouant sur la controverse en plaçant l'acteur face à des contradictions apparentes (par exemple : "Ce qui est étonnant, c'est que tu ne lui dis pas clairement comme tu me le dis à moi que ce n'est pas satisfaisant?»), le chercheur a ensuite invité l'acteur à justifier le jugement préalablement porté. Enfin, il a invité l'acteur à préciser les résultats attendus quant aux actions observées (par exemple : «Et là qu'attends-tu? »).

\subsection{Traitement des données}

Pour formaliser les règles apprises et/ou suivies par les différents acteurs au cours du dispositif expérimental, mais aussi reconstituer la trajectoire de satisfaction des EN, la procédure proposée par Chaliès, Bruno, Méard et Bertone (2010) a été adoptée puis adaptée avec l'ajout d'une cinquième étape de traitement relative à la construction du couplage activité(s) - satisfaction. Au final, les données recueillies ont été traitées en six étapes successives.

1. L'ensemble des ECP et des EAC a été retranscrit verbatim puis découpé en unités d'interaction. Ces unités ont été délimitées à partir de l'objet des significations attribuées par l'acteur autoconfronté aux actions et/ou événements visionnés.

2. Pour chaque unité d'interaction, les éléments d'étayage de la signification attribuée par l'acteur autoconfronté ont ensuite été identifiés. Ces éléments d'étayage correspondent à l'ensemble des circonstances évoquées par l'acteur pour expliquer la façon de s'y prendre pour signifier les événements de la situation de formation visionnés. Ils ont permis de documenter précisément les significations de l'acteur et de lever les éventuels malentendus empêchant au chercheur de signifier de la même façon que lui les événements en question.

3. Pour chaque unité d'interaction, la règle suivie par l'acteur pour signifier son activité et en juger a été formalisée. Par convention, chaque règle a été formalisée à partir de (a) l'objet de la signification attribuée par l'acteur, (b) de l'ensemble des circonstances évoquées par l'acteur pour étayer cette signification et (c) des résultats constatés et/ou attendus. Dans sa forme, chaque règle a été finalement présentée ainsi : [ Objet de la signification " vaut dans les circonstances où «ensemble des circonstances évoquées pour étayer la signification » ce qui obtient comme résultat "ensemble des résultats constatés et/ou attendus»]. Afin de minimiser les interprétations du chercheur, chacune des règles a été étiquetée au plus près du vocabulaire des acteurs.

4. Deux enquêtes grammaticales, dites diachronique et synchronique, ont ensuite été menées. L'enquête grammaticale diachronique a été réalisée pour retracer l'historicité des règles apprises et/ou suivies par les acteurs sur l'ensemble des quatre visites de formation et/ou des leçons. Une enquête grammaticale synchronique a aussi été réalisée. Elle a consisté à comparer les règles suivies par chacun des acteurs lors d'un même temps de formation. Par 
convention, il a été considéré que les acteurs suivaient la même règle si l'objet du jugement, certains éléments d'étayage et les résultats y étant associés étaient identiques.

- Cette cinquième étape a consisté à formaliser le couplage activité - satisfaction. Pour ce faire le traitement engagé a consisté à reconstruire la trajectoire de satisfaction de l'EN. Pour ce faire, trois sous étapes ont été respectées (Figure 1):

- À partir de la nature du jugement (de satisfaction ou d'insatisfaction) porté sur son activité par l'EN, chaque point de la trajectoire de satisfaction a été identifié. Autrement dit, pour chaque règle suivie un point de trajectoire relevant d'un jugement de satisfaction ou d'insatisfaction a été construit. L'enquête diachronique a permis de constituer la trajectoire à partir de ces différents points de trajectoire ;

- Des "phases» ont été instituées dans la trajectoire de satisfaction. Elles ont été construites entre les points de trajectoire préalablement identifiés. Par convention, chaque entrée ou sortie de phase a été établie à partir du passage d'un jugement de satisfaction à un jugement d'insatisfaction ou inversement ;

- Pour chacune des phases de trajectoire construites, le «travail de trajectoire » a enfin été étudié. Pour ce faire, l'activité des acteurs impliqués dans la situation a été étudiée par l'intermédiaire de la reconstitution des règles suivies. L'enquête grammaticale synchronique a permis de mettre en tension les activités individuelles au sein de la situation collective ;

1. La validité des résultats obtenus a été testée. L'ensemble du corpus a été analysé de façon séparée par deux chercheurs. Tous les résultats obtenus ont ensuite été comparés et discutés par les deux chercheurs jusqu'à l'obtention d'un accord. Sur l'ensemble du corpus analysé, moins de $5 \%$ des éléments identifiés ont été source de désaccord entre les chercheurs. Ils ont été rejetés.

\section{Résultats}

Afin d'illustrer par des données empiriques le caractère heuristique de la conceptualisation théorique et des choix de méthode préalablement détaillés pour appréhender le couplage activité(s) - satisfaction, les trajectoires des deux EN impliquées dans le dispositif de formation sont en premier lieu illustrées (Figure 2). Deux phases sont communes aux trajectoires de chacune des deux EN. Elles seront détaillées pour l'une des deux EN (EN1). La dernière phase étant différente pour les deux EN, elle sera détaillée séparément pour chacun des deux EN (EN1 et EN2).

\subsection{Présentation des trajectoires de satisfaction}


Figure 2 : Illustration des trajectoires de satisfaction. Figure 2 : Illustration of a satisfaction trajectory

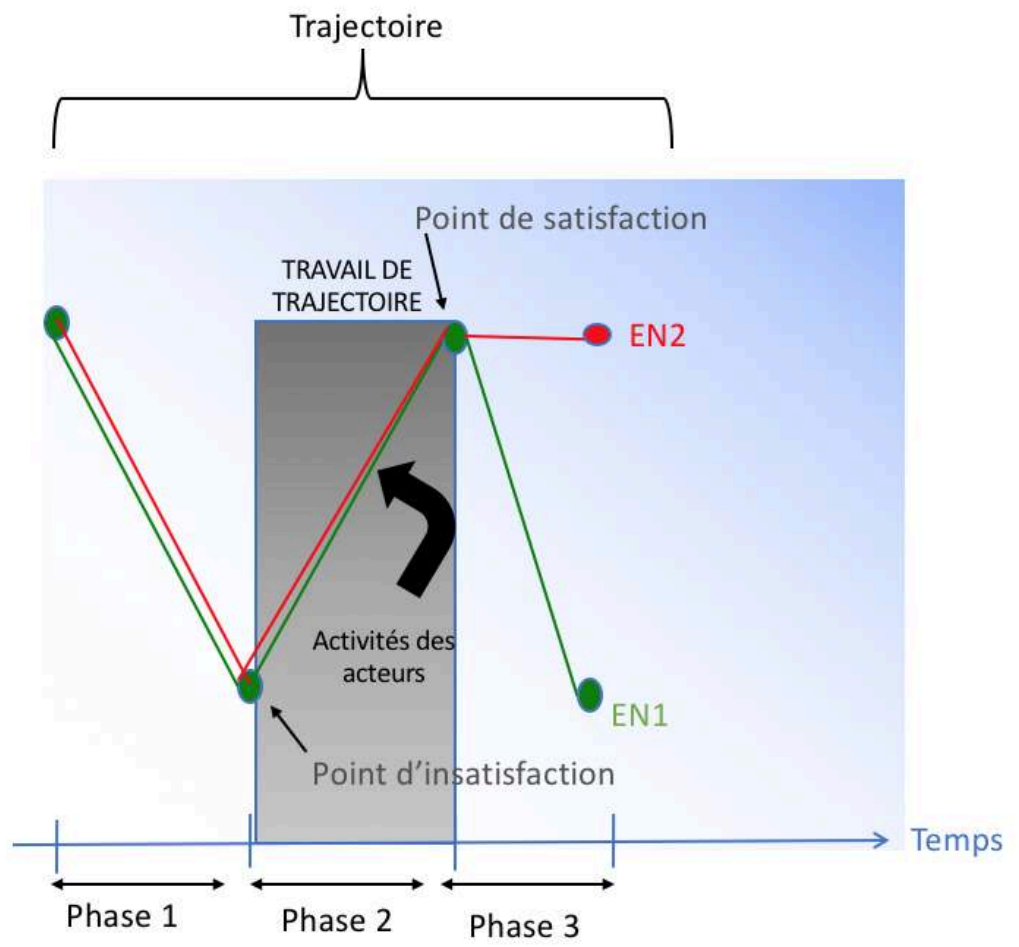

24 La Figure 2 illustre les trajectoires de satisfaction des deux EN (EN1 et EN2). Cette présentation des trajectoires de satisfaction constitue une grille de lecture pour les Phases 1, 2 et 3 détaillées en suivant.

Les deux premières phases sont communes à l'EN1 et à l'EN2. La Phase 3 est différenciée pour les deux EN (EN1 et EN2).

Lors de la Phase 1, la trajectoire de satisfaction des EN rend compte du passage d'un jugement de satisfaction à un jugement d'insatisfaction. Inversement, en Phase 2 la trajectoire de satisfaction rend compte du passage d'un jugement d'insatisfaction à un jugement de satisfaction. Enfin en Phase 3, la trajectoire de satisfaction de l'EN1 rend à nouveau compte du passage d'un jugement de satisfaction à un jugement d'insatisfaction alors que pour l'EN2, la trajectoire rend compte d'une stabilité du jugement de satisfaction.

Toutefois, cette description ne rend pas compte des circonstances de formation ayant contribué (ou non) à la satisfaction ou l'insatisfaction des EN. Ainsi, une étude qualitative de ces circonstances est proposée pour la trajectoire de l'EN1 (Phases 1 et 2) puis pour l'EN1 et EN2 (Phase 3). Pour chacune de ces trois phases, le travail de trajectoire est étudié à partir de la mise en tension des activités de chacun des acteurs impliqués dans les situations. 


\subsection{Présentation qualitative des phases de la trajectoire de satisfaction}

\subsubsection{Phase 1 de la trajectoire de satisfaction}

'EPS, l'EN1 travaille avec ses élèves dans l'activité demi-fond. À l'instant considéré, elle organise les conditions de réalisation de la tâche qu'elle vient de présenter aux élèves. Elle leur précise leur répartition au sein de groupes de travail qu'elle a préalablement envisagés selon leurs niveaux de pratique physique. Tout en répondant aux questions posées par les élèves, l'EN1 leur distribue des chasubles de couleurs différentes et les place dans l'espace de travail. Lorsque tous les groupes sont positionnés, elle procède à leur mise en activité successive par un coup de sifflet (Extrait 1).

Extrait 1 - Extrait des interactions en classe

EN1 : Le départ des (élèves du groupe) verts et des noirs, se fait du plot là-bas (l'EN1

montre les plots)...

Élèves : On fait tous les tours ensemble?

EN1: On fait un tour à la fois, ça vous permet de récupérer...

Élèves : On part ensemble?

EN1: Oui, tout le groupe commence simultanément. Mais les groupes un après

l'autre.

Lors de son autoconfrontation à cet extrait de la leçon, l'EN1 précise au chercheur qu'elle juge son activité plutôt satisfaisante. Elle considère qu'elle s'y prend plutôt «bien » pour lancer les élèves dans la tâche. Selon elle, chaque équipe « sait », en effet, "où elle doit se trouver" avant de s'engager dans la tâche préalablement présentée (Extrait 2).

\section{Extrait 2 - Extrait d'EAC de l'EN}

EN1 : Là, j'essaie de les lancer dans l'activité.

Chercheur $(\mathrm{CH})$ : Les lancer?

EN1 : Oui... en les plaçant sur le lieu de départ.

$\mathrm{CH}:$ Qu'en penses-tu?

EN1 : Là pour moi le lancement est plutôt bien. L'important c'est que chaque équipe

sache où elle doit être avant de se mettre au travail.

À cet instant de l'EAC, l'EN1 suit la règle [«Lancer les élèves en activité » vaut pour «les placer » ce qui obtient comme résultat que chaque équipe «sache où elle doit être avant de se mettre au travail»] pour signifier son activité. Elle juge cette dernière comme satisfaisante. En effet, selon elle, «le lancement est plutôt bien ». Cette satisfaction nait sans doute du fait qu'elle constate les résultats attendus suite au suivi de cette règle, en l'occurrence ici que les élèves "sachent» où se trouver avant de s'engager dans la réalisation de la tâche.

La fin de cette Phase 1 dans la trajectoire de satisfaction advient rapidement puisque, lors de l'ECP1 faisant suite à la leçon, l'EN1 est amenée à associer un jugement d'insatisfaction à son activité de classe. Lors de cet entretien, les formateurs remettent 
en effet en question le jugement de satisfaction qu'elle avait pu initialement associer à son activité de "lancement des élèves dans la réalisation de la tâche». Pour ce faire, ils l'interpellent sur la durée, selon eux beaucoup trop longue, de cette activité (Extrait 3).

\section{Extrait 3 - Interactions lors de l'ECP1}

FU1 : Là, j'ai noté le temps. Fin d'échauffement, 14 h 05. 14 h 05-14 h 10, explication de la tâche 1 . Le début des premières six minutes de course, c'est $14 \mathrm{~h} 13$. Il y a eu huit minutes. Je pense qu'il faut le réduire. Et il y a trois minutes entre 10 et 13 avant que l'on ne voit les premiers élèves courir. Moi là je me dis : « mais que s'est-il passé pendant ces trois minutes?». Normalement, dans la seconde qui suit, ça court.

$\mathrm{T} 1$ : Est-ce que toi tu avais une idée du temps que ce lancement t'avait pris? EN1 : Non... Pas du tout.

La confrontation de l'EN1 à cet extrait d'ECP1 permet de situer combien elle prend conscience, par l'intermédiaire des interventions des formateurs, du «décalage » qui existe entre ce qu'elle pouvait penser initialement de sa mise en activité des élèves et ce qui s'était réellement passé en cours de leçon (Extrait 4).

\section{Extrait 4 - Extrait d'EAC de l'EN1}

$\mathrm{CH}$ : Là tu fais quoi à ce moment de l'entretien?

EN1 : Là, je me dis que j'étais à côté de la réalité. Au début, quand on me demande comment s'est passé le lancement, je trouve justement qu'ils (les élèves) ont eu un temps de pratique plutôt pas mal donc qu'il était bon. Et puis là, ils énumèrent en fait tous les temps pendant lesquels les élèves n'ont pas pratiqué. C. (la T) me demande si j'ai une idée du temps passé justement au lancement... En fait, ce n'était pas du tout ce que je pensais.

$\mathrm{CH}$ : D'accord et donc par rapport au jugement que tu associais à cette activité ?

EN1: En fait, là je découvre en fait et je suis déçue. Je ne suis pas déçue de les entendre. Je suis déçue de voir qu'il y a un décalage entre ce que je pensais et ce qui a vraiment eu lieu dans mon cours.

$\mathrm{CH}:$ Et ton jugement évolue?

EN1: Oui complètement, là je comprends que le lancement ne va pas en réalité. Ce n'est plus satisfaisant.

Lors de cet extrait d'autoconfrontation, l'EN1 suit la règle ["Être à côté de la réalité " vaut pour "découvrir un décalage entre ce que je pensais et ce qui a vraiment lieu " ce qui obtient comme résultat "d'être déçue »] pour signifier son activité. Elle perçoit donc qu'il existe "un décalage » entre ce qu'elle pensait avoir bien fait et "la réalité». Elle s'en trouve "déçue " et associe in fine un jugement d'insatisfaction (" ce n'est plus satisfaisant ») à son activité de lancement des élèves dans la tâche.

Finalement, lors de la Phase 1, la trajectoire de satisfaction de l'EN1 rend compte du passage d'un jugement de satisfaction à un jugement d'insatisfaction porté par l'EN1 sur son activité de «lancement des élèves en activité ».

\subsubsection{Travail de trajectoire en cours de Phase 1 : des activités pour " dé-satisfaire "}

Pour saisir la dynamique intrinsèque de la trajectoire de satisfaction de l'EN1, le travail de trajectoire, c'est-à-dire la nature de l'activité des acteurs engagés dans la formation et leurs mises en tension, est étudié ci-après.

Lors de la situation de formation se situant en Phase 1 de la trajectoire de satisfaction de l'EN1 (ECP1), les formateurs s'attachent à « dé-satisfaire » l'EN1 pour pouvoir ensuite engager une action de formation. Ils s'efforcent, en effet, à remettre en question le jugement de satisfaction porté par l'EN1 sur son activité de classe. Pour ce faire, le FU1 
rend plus précisément compte d'éléments observés lors de cette dernière. Il précise ainsi à l'EN1 le temps de chacune de ses activités auprès des élèves. Il insiste notamment sur la durée ( « il y a eu huit minutes ») du lancement de la situation et lui fait part de la nécessité de « réduire ce temps ». Parallèlement, la T1 s'associe pour ainsi dire à l'activité du FU1, en interrogeant l'EN1 sur ce qu'elle pense des constats faits par le FU (Extrait 3).

Lors de son autoconfrontation à cet extrait de l'ECP1, le FU1 précise au chercheur qui l'interroge qu'il cherche, tout à la fois, à «persuader l'EN» que le problème qu'ils ont préalablement délimité nécessite d'être abordé tout en essayant de ne pas la «mettre en difficulté ». Pour ce faire, il s'efforce « d'amener des preuves » issues de son observation de la leçon. Celles-ci sont relatives au temps consacré par l'EN1 à lancer ses élèves en activité suite à la présentation de la tâche (Extrait 5).

Extrait 5 - Extrait d'EAC du FU

FU1 : J'attire son attention en lui disant que ce n'est pas très performant.

$\mathrm{CH}: \mathrm{Tu}$ attires son attention?

FU1: Oui, pour elle, c'est normal qu'elle perde du temps puisqu'elle doit placer les

élèves... Donc il n'y a aucune raison de changer... Donc je cherche à la persuader que

cela pose problème sans la déstabiliser...

$\mathrm{CH}$ : Lui dire, mais sans la mettre en difficulté?

FU1: Oui, c'est pour cela que j'amène des preuves que j'avais notées pour la persuader qu'il y a vraiment du temps perdu...

$\mathrm{CH}:$ Et votre attente là?

FU1 : Qu'elle se rende compte... et la convaincre de travailler ce point.

Lors de cet extrait de son autoconfrontation, le FU1 suit la règle ["Attirer l'attention de l'EN » sur le caractère inadapté de son lancement de la tâche vaut pour lui « dire que ce n'est pas très performant » et «amener des preuves notées » lors de la leçon ce qui obtient comme résultats qu'elle "se rende compte du problème » et de "la convaincre de travailler " pour y répondre] pour signifier son activité. Le FU1 est donc engagé dans une activité de formation relativement complexe. Il suppose, en effet, que l'EN1 considère que "c'est normal de perdre du temps » à ce moment de la leçon et, qu'en ce sens, elle ne verra pas complètement l'intérêt de changer de façon de procéder s'il ne fait que le lui proposer. Pour surmonter cette difficulté, le FU1 se voit donc contraint « d'amener des preuves » précises, c'est-à-dire «notées » en cours de leçon. Il cherche alors à persuader l'EN1 par des faits relevés qu'il y a «vraiment eu du temps perdu» durant la leçon. Il mène donc une activité de formation avec précautions. Il livre pour ainsi dire certaines preuves concrètes à l'EN1 pour la convaincre du caractère inadéquat de son activité de lancement des élèves dans la tâche tout en s'efforçant de ne pas trop la déstabiliser.

En s'engageant dans ce type d'activité de formation aux côtés de l'EN1, les formateurs contribuent finalement à un travail de trajectoire singulier à l'origine du passage d'un jugement de satisfaction à un jugement d'insatisfaction porté par l'EN1 sur son activité de «lancement des élèves en activité ».

\subsubsection{Phase 2 de la trajectoire de satisfaction}

41 La Phase 2 de la trajectoire de satisfaction de l'EN1 puis le travail de trajectoire réalisé par les acteurs au cours de celle-ci sont successivement analysés ci-après. 


\subsubsection{Phase 2 : d'un jugement d'insatisfaction à un jugement de satisfaction} satisfaction de l'EN1 s'ouvre lors de l'ECP1 et couvre ensuite la Leçon 2. À l'instant considéré de cette leçon, l'EN1 s'engage à nouveau dans une activité de «lancement des élèves en activité». Plus en détail, en fin de délivrance des consignes, l'EN1 leur précise leur répartition au sein de nouveaux groupes de travail. De façon similaire à la Leçon 1 , elle utilise des dossards de couleurs différentes pour constituer ces derniers. Une fois cette répartition effectuée, elle communique ensuite à chaque groupe son plot de départ. Elle s'adresse aux "jaunes" puis aux «noirs", aux "verts» et enfin aux « violets » tout en les maintenant assis face à elle (Extrait 6).

\section{Extrait 6 - Extrait des interactions en classe}

EN1: Quand je vous le dirai, les rouges, vous partez de ce plot jaune (montre le plot). Les blancs, de l'autre là-bas (montre le plot). Les noirs, de ce plot jaune là-bas (montre le plot).

Élèves : (les élèves dont l'équipe a été nommée commencent à se lever)

EN1 : Attendez. Je finis de vous présenter l'organisation. Donc les verts, du gros plot jaune (montre le plot)... Les violets d'ici. Une fois que vous serez placés, je lancerai le départ en sifflant. Dès que je siffle, les groupes commencent tous ensemble à courir. Bon, allez-y.

Élèves : (Les élèves se lèvent et se dirigent vers les plots).

Lors de son autoconfrontation à ce temps de classe, l'EN1 précise au chercheur qu'elle est satisfaite de son activité de lancement des élèves dans la tâche. Selon elle, par la procédure utilisée qui consiste à "garder tous les élèves", s'assurer qu'ils "savent où ils doivent aller» est «mieux que ce qu'elle faisait». En effet, selon l'EN1, les élèves «ne commencent pas » sans savoir ce qu'il y a «à faire et où se placer pour le faire ». Elle trouve finalement cette nouvelle façon de procéder bien «plus efficace » que ce qu'elle faisait initialement (Extrait 7).

\section{Extrait 7 - Extrait d'EAC de l'EN1}

$\mathrm{CH}:$ Qu'est-ce que tu fais là?

EN1: Je les lance dans l'activité. Avant de les envoyer se placer je précise bien ce qu'il y a à faire et où se placer pour le faire.

$\mathrm{CH}:$ Qu'est-ce que tu en penses?

EN1 : C'est bien plus efficace... Par rapport à ce que j'avais fait la dernière fois et ce sur quoi ils (les formateurs) étaient revenus.

$\mathrm{CH}$ : Plus efficace?

EN1: Oui. Là je les garde tous avec moi et ce n'est que lorsque j'ai tout donné qu'ils sont ensuite invités à aller se placer. Ensuite quand je leur dis $\mathrm{OK}$ alors ils y vont immédiatement sans perte de temps et s'engagent vite dans le travail en fait.

$\mathrm{CH}$ : Donc tu es satisfaite?

EN1 : Oui

Lors de cet extrait d'autoconfrontation, l'EN1 suit la règle [«Lancer l'activité » vaut pour " garder les élèves près de soi », leur « délivrer l'ensemble des consignes sur ce qu'il y a à faire et où se placer pour le faire » et « les envoyer se placer une fois toutes les consignes délivrées » ce qui obtient comme résultats que « les élèves se placent sans perdre de temps et s'engagent rapidement dans le travail »] pour signifier son activité. Elle se montre par ailleurs satisfaite de ce qu'elle a pu mettre en place pour optimiser ce moment de la leçon. À ce niveau de développement, il est intéressant de souligner que l'EN1 suit à cet instant une règle que les formateurs lui ont enseignée et expliquée préalablement. En constatant que les résultats souhaités par le suivi de cette règle sont 
effectivement obtenus lors de la leçon, l'EN1 s'en trouve donc finalement satisfaite. L'étude du travail de trajectoire durant cette Phase 2 permet de confirmer ce résultat.

\subsubsection{Travail de trajectoire en cours de Phase 2 : des activités pour " re-satisfaire " l'EN1}

'ECP1 dans une activité d'explication ostensive. Ils associent en effet un nouve exemple à l'activité de classe de l'EN1 nommée, «Lancer les élèves dans la tâche " (étiquette de la Règle). Située au plus près des circonstances de classe de l'EN1, cette nouvelle façon de faire devrait lui permettre de perdre moins de temps (Résultats attendus). Ils lui proposent plus précisément "d'organiser » le matériel nécessaire à la réalisation de la tâche par les élèves et de leur « montrer » comment l'exploiter. Une fois cette présentation effectuée avec tous les élèves rassemblés, ils l'invitent alors à les envoyer simultanément dans l'espace de travail et les lancer dans la tâche sans perdre de temps (Extrait 8).

\section{Extrait 8 - Extrait des interactions lors de l'ECP1}

FU : Tu ne vas pas réduire le temps passé à cela à zéro, mais tu vas le réduire. Donc, ils (les élèves) sont assis. Qu'est-ce que l'on pourrait faire sur ces maillots ? Pour les maillots, tu avais huit équipes, tu pouvais avoir des maillots disposés, parce qu'en plus c'était au sec. Tu aurais pu leur dire : «bon, maintenant, vous allez vous ranger devant les maillots. Vous allez les enfiler et vous allez rester là ». Donc en fait si tu veux l'idée c'est de leur expliquer comment c'est organisé. Puis tu leur expliques comment ça va se passer. Là tu leur montres le déroulement.

T1 : En les ayant tous devant toi... Rangés par exemple en colonnes...

FU : Voilà et tu leur dis « Voyez ce que je vous demande ». Et seulement quand tout ça est calé alors tu les envoies. Et ils commencent quand tu leur demandes.

Autoconfronté à cet extrait de l'ECP1, le FU1 précise au chercheur qu'il «donne un exemple de procédure " envisageable par l'EN1 «dans cette leçon-là». Il explique qu'il prend soin de donner un exemple qui s'enracine dans la pratique de l'EN1 (Extrait 9).

Extrait 9 - Extrait d'EAC du FU

$\mathrm{CH}: \mathrm{Qu}$ 'est-ce que tu es en train de faire?

FU1: Je suis en train de donner un exemple de procédure envisageable sur cette leçon-là.

$\mathrm{CH}:$ C'est-à-dire?

FU1: Je cherche en fait à lui montrer que c'était possible de faire ce qui nous manque en fait... Et que cela aurait permis de gagner quelques minutes. Et qu'elle l'accepte en fait.

$\mathrm{CH}:$ D'accord et là...

FU1 : Là, j'y passe du temps parce que ce qu'on lui propose c'est le contraire de ce qu'elle a fait. C'est-à-dire, elle a dit aux élèves de venir pendant qu'elle organisait et eux au contraire ils se sont éparpillés. Elle a donc été obligée de les rappeler, de redire... Elle a perdu beaucoup de temps.

$\mathrm{CH}$ : Donc là donner un exemple de procédure, ça correspond à quoi ici ?

FU1: C'est lui faire une proposition d'une autre façon de faire en tenant compte de son contexte. C'est donc le concrétiser en fait pour lui montrer que c'est possible et qu'elle accepte de le faire la leçon prochaine.

$\mathrm{CH}: \mathrm{Tu}$ insistes tout de même. Ce n'est pas qu'une proposition en fait?

FU1: Oui, c'est vrai. Je suis plutôt insistant. Parce que je pense qu'elle a tout à 
gagner à faire ce qu'on lui propose. Elle y gagnera même si là ça apparait en contradiction avec ce qu'elle fait.

$\mathrm{CH}$ : Mais là quand même tu... ?

FU1 : Oui j'insiste là. Comme je te le disais, elle n'a pas trop envie de changer cela. Mais nous (avec la T), on a en discuté tous les deux et on est arrivé à se dire qu'en fin de compte ça ne marche pas trop. C'est ce qui m'a convaincu du bien-fondé de ce que je lui propose là.

Le FU1 suit la règle ["Donner un exemple de procédure envisageable» vaut pour "concrétiser » la proposition en fonction du contexte de classe de l'EN1 ce qui obtient comme résultats de lui «montrer que c'est possible» et l'inviter à «le faire lors de la prochaine leçon »] pour signifier son activité. Il agit avec précaution et prend le temps de bien convaincre l'EN1 du caractère envisageable de sa proposition. Il a en effet conscience du fait que l'EN1 "n'a pas trop envie de changer » et que ce qu'il lui propose peut lui apparaitre comme « en contradiction avec ce qu'elle faisait jusqu'ici». Il prend donc le temps de bien en justifier la faisabilité en l'ancrant dans les circonstances de classe de l'EN1. En procédant de la sorte, il s'attend à « convaincre " l'EN1 et, au final, qu'elle «accepte » d'essayer de mettre en œuvre cette nouvelle procédure avec laquelle « elle a tout à gagner» lors de la prochaine leçon. Son engagement dans cette activité de formation est d'autant « insistant » que des échanges menés en amont avec la T1 «l'ont convaincu du bien-fondé de sa proposition ».

Parallèlement, la T1 précise au chercheur que son intervention vise à compléter les propositions du FU. Elle ne fait donc que « redoubler» ce que ce dernier a déjà avancé (Extrait 10).

\section{Extrait 10 - Extrait d'EAC de la T}

$\mathrm{T} 1$ : Là je redouble ce que dit $\mathrm{J}$ (le FU).

$\mathrm{CH}:$ C'est-à-dire?

T1 : Je précise encore plus ce qu'il dit. J'essaye d'aller plus loin. Mais nous en avons discuté avant ensemble donc c'est complémentaire.

$\mathrm{CH}$ : Tu précises et tu vas plus loin, c'est ça?

$\mathrm{T} 1$ : Oui c'est important. Je complète là... Elle (l'EN) peut donc juger elle-même si c'est faisable ou complètement (geste pour signifier une idée irréaliste).

$\mathrm{CH}$ : C'est ce que tu cherches donc... lui montrer que c'est possible?

$\mathrm{T} 1:$ (...) Avec J. (le FU1), on lui dit «tu aurais pu faire autrement, comme ceci et cela »... Et donc, oui là j'imagine qu'elle s'interroge et qu'elle a besoin de rapidement voir si c'est faisable ou pas.

50 Lors de cet extrait d'EAC, la T1 signifie son activité en suivant la règle ["Redoubler le conseil du FU» vaut pour "préciser ce conseil et le compléter» ce qui obtient comme résultat de "permettre à l'EN d'envisager rapidement sa faisabilité »]. Elle s'engage dans une activité "complémentaire " à celle du FU1, car ils ont pu "en discuter ensemble avant " d'entrer dans l'ECP. Son activité lui parait d'autant plus importante qu'elle considère que l'EN1 « s'interroge » sur la faisabilité de ce que le FU1 vient de lui proposer et qu'elle a besoin de "rapidement voir » si cette nouvelle procédure est envisageable ou pas. d'autant plus utile et exploitable («le mettre en ceuvre assez facilement») qu'il est « concret » (Extrait 11).

\section{Extrait 11 - Extrait d'EAC de l'EN1}

$\mathrm{CH}:$ Qu'est-ce que tu fais à cet instant de l'entretien?

EN1 : Il y a beaucoup d'informations. J'essaie de visualiser si ce qu'ils me proposent

peut se faire dans ma classe.

$\mathrm{CH}$ : Visualiser, c'est-à-dire? 
EN1 : Se projeter en fait... Voir si ce qu'ils me disent est jouable ou pas directement dans ma leçon. Si je peux y arriver.

$\mathrm{CH}:$ Et donc là?

EN1 : Là pour moi c'est un bon conseil. C'est très concret donc en fait je vois vite que ce qu'ils me disent de faire colle bien avec ma leçon.

$\mathrm{CH}:$ Et tu t'attends à quoi alors?

EN1 : L'utiliser dès la prochaine fois...

52 L'EN1 suit la règle [« Essayer de visualiser si ce qui est proposé est exploitable » vaut pour «se projeter en essayant de voir si c'est jouable ou pas » ce qui obtient comme résultat de «l'utiliser dès la prochaine leçon »] pour signifier son activité. Selon elle, à cet instant « il y a beaucoup d'informations » qui circulent dans les échanges avec les formateurs. Malgré tout, les formateurs parviennent à l'aider. Elle considère, en effet, que le conseil délivré est « concret » ce qui lui permet de «voir vite que ce que ce qui lui est proposé colle bien avec sa leçon ». Il lui semble au final possible de "l'utiliser dès la prochaine fois $"$.

Lors de la leçon suivante (Leçon 2), l'EN1 est à nouveau confrontée à une situation de lancement des élèves dans une nouvelle tâche. Dans le prolongement de ce qu'elle avait engagé depuis le début de son cycle d'enseignement, elle répartit les élèves par groupe de niveau et leur attribue une couleur de dossard. Après avoir délivré à tous les élèves ressemblés devant elle les principales consignes de la situation, l'EN1 les lance en activité groupe après groupe (Extrait 12)

Extrait 12 - Extrait des interactions en cours de Leçon 2

EN1: Vous avez compris ce qu'il y a à faire? On respecte bien ce que je viens de demander...

Élèves : Oui.

EN1 : Donc maintenant comment on s'organise. Quand je vous le dirai, les rouges, vous partez du plot jaune. Les blancs, de l'autre là-bas (montre). Les noirs, de ce plot jaune là-bas.

Élèves : (les élèves dont l'équipe a été nommée commencent à se lever)

EN1 : Attendez. Les verts vous du gros plot jaune là-bas. Chaque équipe part d'un plot précis et on tourne dans le sens des aiguilles d'une montre. Attention les groupes partent en décalé. OK. Donc maintenant vous vous placez sur vos plots et quand je siffle, vous commencerez à trottiner.

Élèves : (les élèves se lèvent et se dirigent vers les plots).

Lorsqu'elle est autoconfrontée à cet extrait de leçon, l'EN1 précise au chercheur qu'elle juge son activité comme "plus efficace ». Elle suit alors la règle ["Lancer les élèves dans l'activité » vaut pour leur "préciser comment va s'effectuer le travail et son organisation » avant même de les envoyer se placer ce qui obtient comme résultats qu'ils « se placent immédiatement » et «se mettent en activité sans perdre de temps »] (Extrait 13).

\section{Extrait 13 - Extrait d'EAC de l'EN1}

EN1 : Là je les lance dans l'activité. Je précise comment ils vont de voir travailler puis l'organisation. J'insiste là sur où se placer pour démarrer.

$\mathrm{C}:$ Qu'est-ce que tu en penses?

EN1 : C'est plus efficace.

$\mathrm{C}:$ Plus efficace que?

EN1: Que ce que j'ai fait la leçon d'avant. Là comme on a pu le voir ils (les élèves) savent là où ils doivent aller. J'ai organisé, je les oriente et ils y vont immédiatement. On peut donc ensuite se mettre en activité sans perte de temps...

$\mathrm{C}:$ Et ici en termes de satisfaction?

EN1 : Là je suis satisfaite de ce que j'ai fait. 
de son EAC, l'EN1 rend par ailleurs compte du fait qu'elle a pu par elle-même constater le résultat attendu associé à la règle que lui avaient préalablement enseignée et expliquée les formateurs. Elle précise ainsi que son activité de lancement a été « plus efficace ", car elle a permis le placement «immédiat » des élèves dans l'espace de travail et leur engagement dans l'activité "sans perte de temps». Finalement, parvenant à suivre en contexte classe la règle travaillée avec les formateurs, et constatant les résultats y étant associés, l'EN1 s'en trouve donc satisfaite.

\subsubsection{Phase 3 de la trajectoire de satisfaction}

La Phase 3 de la trajectoire de satisfaction de l'EN1 puis le travail de trajectoire réalisé par les acteurs au cours de celle-ci sont successivement analysés ci-après. En suivant, la Phase 3 de la trajectoire de satisfaction de l'EN2 puis le travail de trajectoire réalisé par les acteurs de cette triade sont analysés.

\subsubsection{Phase 3 : d'un jugement de satisfaction à un jugement d'insatisfaction (EN1)}

Ouverte en cours de Leçon 2, la Phase 2 détaillée précédemment couvre ensuite l'ECP2, mais aussi le Leçon 3 et l'ECP3 y faisant suite. La Phase 3 de la trajectoire de satisfaction de l'EN1 s'ouvre quant à elle en Leçon 4. À l'instant considéré de cette leçon, l'EN1 s'engage à nouveau dans une activité de "lancement des élèves en activité ». Toutefois, contrairement à ce qu'elle faisait jusqu'à lors, l'EN1 ne place pas les élèves par groupe sur l'espace de travail. Ayant permis à ces derniers d'évaluer lors des leçons passées leur propre vitesse maximale aérobie (VMA), l'EN1 leur demande de se positionner individuellement en fonction de cette dernière de sorte que leur distance de course soit adaptée à leur capacité. À l'instant considéré, l'EN1 présente à ses élèves regroupés devant elle cette nouvelle modalité d'organisation (Extrait 14).

\section{Extrait 14 - Extrait des interactions lors de la Leçon 4}

EN1: Aujourd'hui c'est un peu différent puisque vous avez vos VMA. On va travailler tout seul. Donc vous allez aller vous placer en fonction de vos VMA. Le premier plot là-bas (Montre) c'est le départ de ceux qui sont à 8 (de VMA), le second là pour ceux à 10, le troisième là-bas (Montre) à 12 et le dernier c'est pour ceux qui ont plus de 12.

Élèves : On n'est plus par équipe alors?

EN1 : Et non. Par niveau aujourd'hui. Chacun va travailler à son niveau pour progresser.

Une élève : Et si on est à 11 Madame ?

$\mathrm{EN}$ : Et bien tu te mets à 12 . Un peu au-dessus. Ça va te faire progresser comme ça... On se place sur le plot du dessus de sa VMA.

Élèves : (Des élèves se lèvent et vont vers les plots. D'autres élèves restent assis et questionnent l'EN). Moi Madame j'étais à 8 mais je peux quand même courir avec Sabrina?

EN1 : On court à sa VMA. Ce ne sont plus les groupes qui comptent..

Élèves : Même si c'est la même VMA

EN 1 : Si... Bon allez en place.

Lorsqu'elle est autoconfrontée à cet extrait de leçon, l'EN1 précise au chercheur qu'elle est «déçue " par ce moment qui la "remet en question ». Elle considère que les élèves " ont été perturbés » par la nouvelle organisation. Selon elle, c'est ce qui explique qu'elle soit retombée dans «ses travers en essayant de répondre à l'un ou à l'autre (élève) » et donc à ne «plus parvenir à gérer le groupe ». Elle a conscience de "perdre énormément de temps" 
alors même qu'elle pensait "avoir bien calé ces phases entre les différentes tâches de la leçon » (Extrait 15).

\section{Extrait 15 - Extrait d'EAC de l'EN1}

EN1 : Là, je l'ai senti pendant la leçon. Je retombe dans mes travers en essayant de répondre à l'un ou à l'autre...

$\mathrm{CH}: \mathrm{Tu}$ es moins satisfaite de ton activité alors?

EN1: Oui je suis déçue là de ce que je fais. En même temps j'ai modifié l'organisation donc ils ont été perturbés. Je leur propose de travailler tout seul en fonction de leur VMA et eux ils sont sur le travail de groupe depuis le début.

$\mathrm{CH}$ : C'est ce qui explique pour toi la difficulté là ?

EN1 : Oui avant je gérais des groupes et là c'est presque une gestion individuelle de mise au travail. C'est pour ça que je perds énormément de temps.

$\mathrm{CH}$ : Donc c'est insatisfaisant même si c'est la première fois que tu...

EN1: Oui parce que je pensais avoir bien calé ces phases entre les tâches, on ne perdait pas de temps et là ça remet pas mal en question.

\subsubsection{Travail de trajectoire en cours de Phase 3 : des activités pour " re-satisfaire "} l'EN1

Contrairement au travail de trajectoire mené en Phase 1 de la trajectoire de satisfaction de l'EN1, les formateurs s'attachent en Phase 3 à « re-satisfaire " l'EN1 pour pouvoir ensuite engager une action de formation. Ils sont en effet « en accord» avec le jugement d'inadéquation porté par l'EN1 sur son activité de lancement des élèves dans la tâche et s'efforcent donc de " profiter de la situation " pour l'engager dans un «enrichissement de sa pratique ». L'extrait suivant tiré de l'ECP 4 est à ce titre très explicite (Extrait 16).

\section{Extrait 16 - Extrait des interactions lors de l'ECP4}

$\mathrm{T} 1$ : Là, tu en penses quoi, après coup du lancement de la dernière tâche ?

EN1 : Pas top. Ils n'ont pas bien compris ce que j'attendais d'eux, je crois en fait.

$\mathrm{T} 1$ : Pas bien compris?

EN1 : J'ai trop modifié l'organisation en fait en leur demandant de travailler seuls à partir de leur VMA.

FU : Oui je pense aussi. Je crois qu'on est d'accord nous aussi sur ce constat.

T1 : Par contre moi je pense qu'il ne faut pas le voir comme un échec là. C'est compliqué tout ce que tu changes. On doit essayer de profiter de ce qui s'est passé pour que tu parviennes en fait à enrichir ta pratique.

FU : Oui tu modifies beaucoup d'un coup donc c'est normal qu'on retombe sur le problème du temps passé à présenter et à engager les élèves dans l'activité.

$\mathrm{T} 1$ : On doit essayer en fait de trouver un moyen de toujours réduire le temps, mais peut-être en modifiant un peu ce que tu faisais.

Lors de son autoconfrontation à cet extrait de l'ECP4, la T1 précise au chercheur qui l'interroge qu'elle cherche à faire en sorte que «l'EN ne baisse pas les bras». Elle a en effet conscience du fait qu'elle rencontre une «situation difficile». Selon elle, l'EN1 a "fait beaucoup d'efforts pour essayer de stabiliser une façon de faire efficace " et là tout doit lui sembler remis en question. Elle considère qu'il faut donc "l'encourager » et faire en sorte de rapidement trouver un "moyen d'adapter ce qu'elle faisait pour que cela continue à être efficace » même si elle modifie quelque peu l'organisation de ses tâches (Extrait 17).

Extrait 17 - Extrait d'EAC de la T

$\mathrm{CH}$ : Là, tu interviens davantage là ?

T1: Oui. C'est important pour moi. Là je me dis qu'elle rencontre une situation difficile donc je l'encourage à persévérer.

$\mathrm{CH}:$ C'est-à-dire?

T1 : Il faut se dire qu'elle a fait depuis le début beaucoup d'efforts pour essayer de stabiliser une façon de faire efficace. Donc, il faut l'aider à trouver un moyen 
d'adapter ce qu'elle faisait pour que cela continue à être efficace.

$\mathrm{CH}$ : C'est ce que tu fais là ?

T1 : Oui il faut absolument qu'en sortant elle ne baisse pas les bras. Donc il faut vite qu'on trouve comment aménager un peu ce qu'elle faisait.

61 Lors de cet extrait d'autoconfrontation, la $\mathrm{T} 1$ suit la règle [«Encourager l'EN à persévérer » vaut pour "trouver rapidement un moyen d'adapter ce qu'elle faisait » ce qui obtient comme résultats qu'elle retrouve une efficacité dans sa façon de faire et "ne baisse pas les bras»] pour signifier son activité de formation. Elle considère en effet que ce que vit à cet instant l'EN1 est «difficile » compte tenu de tous les efforts entrepris jusqu'ici pour «stabiliser une façon de faire efficace » et qu'il est donc de sa responsabilité de l'accompagner vers davantage de satisfaction en l'aidant à optimiser sa pratique de classe.

\subsubsection{Phase 3 : d'un jugement de satisfaction à un jugement de satisfaction (EN2)}

62 La trajectoire de satisfaction de l'EN2 s'ouvre lors de la L1. Lors de cette leçon d'EPS, l'EN2 travaille avec ses élèves dans l'activité lutte. Dès l'ECP1, les formateurs s'efforcent de travailler autour de l'objet de formation énoncé de la façon suivante par le FU2 : [ "Faire démontrer par certains élèves ce qui est attendu d'eux" vaut pour " renforcer la verbalisation » ce qui obtient comme résultats « de faire visualiser » et que tous les «élèves (aient) compris »]).

La Phase 3 de la trajectoire de satisfaction de l'EN2 s'ouvre en Leçon 3. À l'instant considéré de cette leçon, l'EN2 s'engage à nouveau dans une activité de démonstration. Après une rapide présentation des consignes d'une tâche dite de la «tortue ", l'EN2 procède à la mise en place d'une démonstration par deux élèves. Lors de cette démonstration, elle aide les deux élèves afin qu'ils parviennent à faire ce qui est attendu d'eux. Pour ce faire, elle répète certaines consignes préalablement délivrées et en apporte de nouvelles (Extrait 18).

\section{Extrait 18 - Extrait de la Leçon 3}

EN2: Vous écoutez. En combat, par moment, il va y avoir ce que moi j'appelle la tortue. L'élève qui va se mettre comme cela (elle mime). Viens ici (elle désigne un élève). Oui, toi (elle désigne un autre élève). Parce que si je te demande de faire ou de répéter ce que j'ai dit... Tu t'assois sur les fesses et tu me regardes Tu te mets en tortue. Les autres, on regarde. Hop, les coudes devant. Donc, regardez l'exercice suivant. Le but va être de renverser son adversaire. Pour renverser son adversaire, comment je vais faire? Je vous montre. Je passe une main au niveau de son coude (mime). L'autre main, au milieu. Regardez. Ma main ressort ici. OK? Je rattrape l'autre main. Du coup, cela s'appelle une saisie. Moi, je vais basculer mon poids du corps sur l'adversaire (elle pousse l'attaquant, le défenseur subit dessous). Voyez, je commence à pousser. Pour pousser, je peux aussi utiliser mes jambes et mes pieds (elle montre les jambes). L'action de mes bras va être de tirer vers moi et de pousser avec mon corps. Et ensuite on continue (les élèves cessent la démonstration). Et on le repose sur les épaules. Le but c'est d'avoir les épaules de mon adversaire sur le dos. (Les élèves partent réaliser la tâche. L'EN2 constate leur réussite). C'est bien...

Lorsqu'elle est autoconfrontée à cet extrait de leçon, l'EN2 met en avant la nécessité d'inclure le temps de démonstration dans la présentation de la tâche pour que ses élèves comprennent mieux ce qui est à faire et comment le faire. Selon elle, le profil de ses élèves, dont certains présentent des difficultés avérées de compréhension des consignes orales, rend pertinent cette activité d'enseignement. Elle souligne en effet 
que ses élèves "ont besoin de l'imaginer (la tâche à faire)" avant de s'engager dans sa réalisation (Extrait 19).

\section{Extrait 19 - Extrait d'EAC de l'EN2}

$\mathrm{CH}:$ Qu'est-ce que tu fais là?

EN2 : Je fais faire une démonstration.

$\mathrm{CH}:$ Qu'est-ce que tu en penses?

EN2: Cela permet d'avoir le visuel en même temps que l'auditif parce que je vais faire démontrer en même temps que je vais expliquer.

$\mathrm{CH}:$ Et tu penses que c'est...

EN2: C'est bien.

$\mathrm{CH}$ : Pourquoi tu penses que c'est bien de faire le visuel et l'auditif?

EN2 : Parce que, eux, au niveau cognitif, ils sont limités. C'est un peu plus difficile, donc ils ont besoin de l'imaginer, de le vivre au moins une fois avant de partir sur la tâche.

$\mathrm{CH}$ : Cela aboutit à quoi que tu démarres sur cette démonstration en la commentant?

EN2 : Après, les lancer dans l'activité et que tout le monde réussisse, au plus vite, et le mieux possible.

L'EN2 suit à alors la règle suivante pour rendre compte de son activité : [ « Faire faire une démonstration aux élèves » vaut pour «faire démontrer et expliquer » c'est-à-dire « donner le visuel en plus de l'auditif » ce qui obtient comme résultat que "les élèves réussissent au plus vite et le mieux possible»]. Elle constate une partie des résultats attendus dans la réalisation des élèves et s'en trouve donc satisfaite ( «c'est bien »). À cet instant, l'EN2 suit donc une règle qui lui a été enseignée lors des temps de formation précédents et constate les résultats associés par les formateurs à celle-ci. Elle constate, plus précisément, que les élèves réussissent mieux et plus vite en étant aidés par une double présentation orale et visuelle de ce qu'il y a à faire.

La fin de la Phase 3 de la trajectoire de satisfaction advient lors de la leçon 4. L'EN2 est à nouveau amenée à associer un jugement satisfaction à son activité de classe. Lors de cette leçon elle présente à ses élèves une nouvelle tâche dans une nouvelle activité physique et sportive, la natation. À l'instant considéré de la leçon, elle leur a précisé les principales consignes pour parvenir à faire ce qui est attendu : une poussée puis une coulée ventrale efficace depuis le bord du bassin. Pour faciliter la compréhension des consignes, elle a pris le soin de les personnaliser en employant la première personne ( «je pousse... je pars... »). Suite à cette présentation, elle a demandé à un élève de réaliser une démonstration de "poussée ventrale». En cours de démonstration, elle a toutefois précisé certaines consignes : "Il va pousser sur ses jambes pour aller s'allonger. Il va tenir » (Extrait 20).

\section{Extrait 20 - Extrait de la Leçon 4}

EN2 : Le but va être de passer à un équilibre horizontal en position ventrale. J'ai la tête dans l'eau, le regard qui vers les carreaux. Quand je suis allongée au maximum, et que je ne bouge plus, je ne coule plus, je repasse en position assise. Je pars en position assise, je pousse sur mes pieds pour aller m'allonger. Quand j'ai réussi à avoir mon allongement maximal, je suis tirée au maximum. Je peux revenir dans cette position... En position assise, semi-debout. Est-ce qu'il y en a qui n'ont pas compris? Tu nous montres la démonstration (désigne un élève). On regarde tous. Regarde là-bas. Il va pousser sur ses jambes pour aller s'allonger. Il va tenir. (l'élève démontre). Oui. Il s'allonge au maximum et il faut essayer de maintenir un peu plus long, la glisse, plus longtemps. Tout le monde prend sa place...

Lors de son EAC relatif à cet extrait de classe, l'EN2 précise qu'elle exploite la démonstration pour permettre aux élèves qui n'ont pas compris les consignes délivrées 
oralement de s'en saisir en voyant ce qu'il y a à faire. Cette démonstration est aussi l'occasion de "redonner des consignes » même si elle juge qu'elle est certainement un peu trop insistante en les répétant à trois reprises (Extrait 21).

\section{Extrait 21 - Extrait d'EAC de l'EN2}

$\mathrm{CH}: .$. Tu fais faire une démonstration, c'est ça?

EN2 : Oui, en redonnant les consignes.

$\mathrm{CH}:$ Comment tu la juges?

EN2 : C'est bon même si répétitif. Il vaut mieux. Je l'ai répété trois fois. (...). C'est bien. Mais, ça veut dire quoi s'allonger plus finalement ? Il y en a un qui aurait pu me le dire. Ça veut dire quoi ? J'aurais pu donner une distance ou...

$\mathrm{CH}:$ Et le résultat de ta démonstration où tu donnes des consignes?

EN2: Faire démontrer, ça permet peut-être à ceux qui ne m'ont pas compris verbalement, en le voyant, de savoir ce qui est fait... et justement de modifier certains comportements, s'allonger un peu plus. Je n'ai pas été très précise, mais...

Lors de son EAC, l'EN2 rend compte de son activité en suivant la règle [" Faire démontrer ce qui est attendu par des élèves » vaut pour « redonner des consignes » et « faire voir » ce qui obtient comme résultats de permettre aux élèves de "voir ce qui n'a pas été compris verbalement" et "de modifier leurs comportements»]. Elle suit donc une règle que les formateurs lui ont enseignée lors de l'ECP1. Par ailleurs, comme le lui ont aussi proposé les formateurs, les consignes sont proposées en termes d'activités d'élèves afin qu'elles soient plus facilement saisissables. Enfin, elle insiste sur le sens de ce qui est demandé en concrétisant par exemple ses attentes: les élèves doivent parvenir à "glisser » sur l'eau après avoir poussé. Au final, elle juge son activité plutôt satisfaisante («c'est bon... c'est bien... »).

Finalement, la Phase 3 de la trajectoire de satisfaction de l'EN2 rend compte d'une stabilité de son jugement de satisfaction sur son activité de délivrance des consignes qui intègre une « démonstration ».

\subsubsection{Travail de trajectoire en cours de Phase 3 : la complémentarité des acteurs}

Pour saisir la dynamique intrinsèque de la trajectoire de satisfaction de l'EN2, le travail de trajectoire est étudié ici détaillé. Lors de l'ECP3 qui a suivi la L3, les formateurs donnent des explications à l'EN2 sur ces démonstrations. Les formateurs ont observé des tentatives de mise en place de démonstrations par les élèves afin d'aboutir à une présentation plus claire et compréhensible des tâches à réaliser. Pour autant, ils évoquent le caractère encore inadéquat des démonstrations de l'EN2. Pour l'aider à surmonter ces difficultés, ils s'engagent donc dans des explications. Le FU2 souligne ainsi de son côté toute l'importance de la clarté et de la cohérence des consignes verbales délivrées en amont de la démonstration pour que celle-ci ait du sens pour les élèves. La T2 propose, quant à elle, à l'EN2 de «se mettre dans la peau de l'élève » afin de mieux préparer les consignes qui précèderont ou accompagneront la démonstration (Extrait 22).

\section{Extrait 22 - Extrait des interactions lors de l'ECP3}

FU2 : Et toi, tu dois vraiment te débattre pour montrer à l'autre si ce qu'il fait c'est efficace. Tu vois? Même à un moment donné, il y en a un qui a dit: "on peut s'échapper?». "Non, on se laisse faire surtout». Si tu veux, c'était contradictoire. Ce que l'on veut dire si tu veux, il y a un truc à clarifier. Il faut que l'on continue...

T2 : Il faut que tu te mettes dans la peau de celui qui immobilise et dans la peau de l'autre, celui qui se défend aussi. Il ne faut pas que tu oublies tous les rôles qui permettent l'utilisation du poids du corps. Quand on est celui qui s'échappe, on 
comprend aussi. Après, quand on change de rôle, on comprend. Ça aide à mieux comprendre.

71 Lors de son EAC, la T2 précise qu'elle insiste, en continuité avec les propos tenus par le FU2 relatifs aux consignes délivrées verbalement, sur la nécessité pour l'EN2 de se mettre «à la place de l'élève » afin qu'elle envisage davantage l'accessibilité de ce qu'elle dit à ses élèves. Selon elle, les consignes sont trop souvent inexploitables, car non accessibles et non utiles pour réaliser ce qu'il y a à faire. La T2 précise, par ailleurs, que c'est là une explication de la règle qu'elle a déjà donnée à l'EN2 lors d'un temps de formation antérieur (Extrait 23).

\section{Extrait 23 - Extrait d'EAC de la T2}

$\mathrm{T} 2$ : Je fais quelque chose que je lui ai déjà fait. Je me mets à la place de l'élève qui écoute. Toujours pour qu'elle s'assure que c'est compréhensible. Et il y a du sens.

$\mathrm{CH}$ : Pour arriver à quel résultat tu joues le rôle de l'élève...

T2 : Là, l'objectif, c'était d'utiliser son poids du corps pour l'immobiliser. C'était pour renforcer cette action d'utilisation du poids.

$\mathrm{CH}$ : Mais toi, pourquoi tu fais exprès, tu joues le rôle de l'élève qui écoute?

T2: Parce que c'était insuffisant... Les élèves ne comprennent pas sinon ce qui est dit et à quoi cela peut leur être utile.

Lors de cet extrait d'EAC, la T2 met en avant le caractère " insatisfaisant » du suivi par l'EN2 de la règle qui lui a été préalablement enseignée («c'est insuffisant »). Elle s'engage dans une activité d'explication visant à proposer un autre exemple. La T2 conseille de délivrer des consignes pour les rendre compréhensibles et exploitables par les élèves tant lors de la démonstration, qui devrait permettre de les voir vivre, que lors des tentatives suivantes. Elle propose alors à l'EN2 de "se mettre à la place de l'élève » comme elle a pu elle-même le faire lors de la leçon ("écouter comme un élève ») afin d'optimiser ses consignes et donc d'accroître l'efficacité de la démonstration.

Le FU2 reconnaît que les solutions proposées par la T2 sont cohérentes. Il les considère pour autant comme « déconnectées » de la dynamique de formation en cours (Extrait 24).

\section{Extrait 24 - Extrait d'EAC du FU2}

FU2: C'est très cohérent, mais c'est déconnecté par rapport à ce que je viens de dire.

$\mathrm{CH}:$ C'est déconnecté, c'est-à-dire?

FU2 : Je suis en train de me dire " Mince, elle a un temps de retard! ». C'est-à-dire par rapport à l'argumentaire que j'étais en train de mener et de l'amener: " on est d'accord, on y va? Oui, allez, c'est parti». On est en train de passer dans la phase de conseil. Elle dit: "et oui, c'est ce que j'avais pensé par rapport à la tâche, il faut que l'autre »... En fait, elle reprécise ce que je disais tout à l'heure. Je me dis oui, c'est cohérent, mais un peu en retard... Je suis une peu inquiet pour l compréhension de M. (l'EN2)

$\mathrm{CH}:$ Pour toi, tu perds du temps?

FU2: Ce n'est pas une perte de temps. C'est plutôt de l'inquiétude en termes de: «Est-ce que M. (l'EN2) comprend ce truc-là ?» Et puis surtout, cela gêne-t-il la compréhension? Cela risque de bousculer ce que j'avais essayé de proposer, alors je ne sais pas si c'était adroit, mais j'ai essayé de clarifier...

Lors de son EAC, le FU2 suit la règle suivante pour rendre compte de son activité : ["Vivre la situation d'entretien comme difficile» vaut pour «constater que la T2 intervient de façon cohérente déconnectée par rapport à l'argumentaire engagé » ce qui obtient comme résultat « d'être inquiet pour la compréhension de l'EN2 »]. Le FU2 estime l'intervention de la T2 cohérente par rapport aux propos, mais décalé temporellement. Il est donc préoccupé par la compréhension de l'EN2. 
Autoconfrontée à ce même extrait, l'EN2 souligne paradoxalement qu'elle apprécie le caractère, à ses yeux, complémentaire et collaboré des activités des deux formateurs. Selon elle, tout l'intérêt de cette situation de formation réside dans le fait que le FU2 initie théoriquement les conseils et que la $\mathrm{T} 2$ se charge ensuite de les concrétiser en essayant de les adapter aux circonstances de sa classe ( $S$. (le FU2) la théorie et O. (la T2) le terrain ») (Extrait 25).

\section{Extrait 25 - Extrait d'EAC de l'EN2}

EN2 : Là, j'ai l'impression qu'O. (la T2) me répète ce que m'a dit S. (le FU2). Mais plus en des termes compréhensibles par les élèves. C'est un peu : S. (le FU2) la théorie et O. (la T2) le terrain. Regarde, à cet endroit-là...

$\mathrm{CH}:$ C'est important pour toi qu'il y ait cette répétition?

EN2 : Oui.

$\mathrm{CH}$ : Pourquoi elle est importante cette répétition?

EN2 : Ce sont des déclinaisons. S. (le FU2) me l'a dit dans des mots que j'entends et que je comprends et qui viennent d'être appuyées par une démonstration, un exemple presque d'O. (la T2) qui est du terrain. J'ai les deux.

$\mathrm{CH}$ : Cela aboutit à quel résultat alors?

EN2 : Cela renforce les propos de S. (le FU2). Et du coup, une meilleure compréhension pour moi.

Lors de cet extrait d'autoconfrontation, l'EN2 précise le fonctionnement de la dyade de formateurs. Selon elle, la $\mathrm{T} 2$ joue un rôle très important en «répétant» les propos du FU2 avec un vocabulaire de terrain moins théorique ce qui, au final, lui permet de mieux comprendre ce qui lui est proposé comme conseil. Pour l'EN2, les activités des formateurs sont donc pour ainsi dire complémentaires. Lors de ce travail de trajectoire, les formateurs mènent donc des activités singulières, mais complémentaires.

\section{Discussion}

L'idée n'est pas ici de proposer une discussion construite à partir d'une généralisation des résultats obtenus dans le cadre d'une étude de cas, et ce même si leur récurrence dans plusieurs autres études menées au sein du programme de recherche pourrait permettre de l'envisager (voir par exemple: Amathieu, Escalié, Bertone, \& Chaliès, 2018; Chaliès, \& Amathieu, 2013). Les résultats préalablement détaillés nécessiteraient en effet d'être validés par des travaux menés avec des échantillons scientifiquement plus significatifs. De la même manière, le contexte de l'enseignement de l'EPS revêt des singularités fortes en matière de formation des EN (par exemple la préoccupation d'aider ces derniers à gérer l'espace de travail souvent bien plus complexe que celui d'une salle de classe) et il serait en ce sens nécessaire de réaliser un tel dispositif de formation auprès d'EN d'autres disciplines scolaires. Enfin, une étude complémentaire pourrait être envisagée pour soumettre aussi à l'étude des moments plus informels de formation (par exemple lors des temps de discussion entre pairs) et leurs retombées sur la satisfaction professionnelle des EN. Dans la continuité de l'objet premier de l'article, cette discussion cherche plutôt à s'appuyer sur les résultats produits pour interroger le caractère heuristique de la conceptualisation théorique et des choix de méthode adoptés afin d'appréhender le couplage activité(s) - satisfaction professionnelle. 


\subsection{Le caractère situé et dynamique de la trajectoire de satisfaction professionnelle des enseignants novices}

78 En permettant de rompre avec une conception encore dominante dans le champ assimilant la satisfaction professionnelle à un état "a situé » par rapport à l'activité professionnelle, la conceptualisation théorique détaillée en amont apparait en premier lieu comme heuristique. C'est ici notre premier point de discussion. À partir de choix singuliers de méthode, cette conceptualisation rend en effet possible la mise en évidence du caractère dynamique de la (trajectoire de) satisfaction des EN tel qu'on peut le retrouver dans certains travaux menés en psychologie du travail (voir par exemple : Schwartz, 2007). C'est ici une prise de position d'autant plus originale dans le champ que bon nombre de travaux, le plus souvent menés depuis des conceptualisations théoriques psychologiques, soutiennent le postulat d'une certaine stabilité de la satisfaction des enseignants (McInerney, Korpershoek, Wang, \& Morin, 2018; Skaalvik, \& Skaalvik, 2017 ; Von der Embse, Sandilos, Perdergast, \& Mankin, 2016).

79 Au contraire, la conceptualisation théorique détaillée au sein de cet article permet de situer combien la satisfaction s'inscrit dans un « couplage intrinsèque " avec l'activité (Chauviré, 2010). Théoriquement, l'EN ne peut effectivement être satisfait de son travail qu'à la double condition d'avoir dans l'activité de classe (i) « suivi des règles » que les formateurs lui ont préalablement enseignées et expliquées et (ii) constater in situ les résultats qui leur sont usuellement associés. Autrement dit, pour être satisfait de son activité, l'EN doit pouvoir déployer sur l'expérience de classe les critères de satisfaction appris antérieurement avec les formateurs, c'est-à-dire les règles objets de formation qui ne sont rien d'autre que les "expériences normatives situées" (Chaliès, 2016) acceptées par la communauté professionnelle et rendant intelligibles et évaluables les activités de chacun de ses (futurs) membres.

Cette conceptualisation comporte ceci d'original qu'elle pose la satisfaction comme le résultat d'une activité de jugement situé de la part de l'acteur dans le cadre de son activité professionnelle c'est-à-dire dans un arrière-plan contextuel toujours particulier (Wittgenstein, 1996). L'expérience vécue par l'acteur au cours de son activité professionnelle lui permet d'exprimer son jugement de satisfaction (Chauviré, 1992). En ce sens, la satisfaction par son caractère situé ne peut être qu'une expérience dynamique au regard de la trajectoire de l'acteur dans son activité professionnelle. Le contexte des activités réalisées évoluant sans cesse, la satisfaction professionnelle nourrie par ces dernières fluctue aussi comme le montrent nos résultats. C'est donc ici une conception "contextualiste» de l'expérience de satisfaction (Travis, 2003) que nous défendons. Ce sont effectivement les circonstances rencontrées en contexte de travail en classe et/ou de formation qui ont en effet permis, ou à l'inverse pas permis, à l'EN de porter un jugement d'appréciation sur ce qu'il pouvait ou avait pu réaliser. Dans certaines circonstances, ne parvenant pas à obtenir les résultats souhaités, l'EN a effectivement porté un jugement d'insatisfaction sur son activité de classe. Dans d'autres circonstances, parvenant à obtenir les résultats attendus, il a au contraire porté un jugement de satisfaction.

81 Cette conceptualisation théorique présente un certain nombre d'intérêts en matière notamment d'aménagement des circonstances de travail et/ou de formation pour justement autoriser les EN à accéder à des épisodes de satisfaction professionnelle. Elle 
permet en effet l'identification de courts épisodes de satisfaction au sein d'une première année d'entrée dans le métier d'enseignant souvent considérée comme difficile à vivre (Borman, \& Dowling, 2008; Gold, 1996) et conduisant certains EN à l'abandon (par exemple, Goldring, Taie, \& Riddles, 2014). La mise en évidence de ces épisodes de satisfaction, certes éphémères et le plus souvent transitoires, invite cependant à relativiser les résultats de plusieurs études concluant à une insatisfaction chronique des EN et une institutionnalisation du mal-être chez les enseignants novices voire expérimentés (Lantheaume, \& Hélou, 2008; Maroy, 2008) en interrogeant l'équilibre dynamique du sujet en situation de travail (Ciavaldini-Cartaut, \& Blaya, 2018). Plus en avant, ils permettent de reconsidérer la formation comme un levier pour sortir les EN d'un jugement d'insatisfaction chronique sur leur activité de classe et, de temps en temps, leur autoriser l'attribution d'un jugement de satisfaction source momentanément d'une amélioration de la qualité de vie au travail (Billaudeau, \& Vercambre-Jacquot, 2015 ; Fotinos, \& Horenstein, 2011).

\subsection{Satisfaction professionnelle et activités : le travail de trajectoire} l'activité d'un acteur avec son (in)satisfaction, ce second point souligne le caractère situé de ce couplage à l'articulation des activités des différents acteurs, formateurs et EN, engagés dans les situations de travail et/ou de formation. La satisfaction est en effet directement dépendante de la nature de cette articulation d'activités.

À partir de l'étude du travail de trajectoire déployé lors de chacune des phases de la trajectoire de satisfaction de l'EN étudiée, les résultats détaillés en amont mettent en évidence la réciprocité existante entre les activités menées par les formateurs lors des ECP et la nature des jugements portés par l'EN sur son activité. En surface, ils montrent précisément comment, accompagnée par le travail des formateurs, l'activité de "lancement des élèves dans la tâche» initialement réalisée par l'EN1 devient progressivement conforme aux attentes de la communauté professionnelle en permettant d'atteindre les résultats souhaités ("les élèves se placent sans perdre de temps et s'engagent rapidement dans le travail ») et, de fait, contributive d'un jugement de satisfaction chez l'EN. En profondeur, grâce au grain (fin) d'analyse adopté dans cette étude, les résultats permettent en outre d'identifier toute la complexité du travail de trajectoire engagé (même s'il dépasse en partie le cadre des extraits proposés), de la trajectoire de satisfaction de l'EN1 et de leurs articulations. Ainsi, en Phase 1, les formateurs s'attachent d'abord à « dé-satisfaire » avec précaution l'EN1 pour pouvoir ensuite l'engager avec une intention partagée dans une activité de formation. Pour ce faire, le FU1 mène une activité singulière qu'il signifie lors de son autoconfrontation en suivant la règle : [ Attirer l'attention de l'EN » sur le caractère inadapté de son lancement de la tâche vaut pour lui "dire que ce n'est pas très performant » et "amener des preuves notées" lors de la leçon ce qui obtient comme résultats qu'elle "se rende compte du problème » et «soit convaincue de travailler » pour y répondre]. Aidé par la T1, il cherche tout à la fois, à persuader l'EN1 que le problème qu'ils ont préalablement délimité nécessite d'être travaillé tout en s'efforçant de ne pas trop la déstabiliser. Cette activité de formation confirme la nécessité pour les formateurs d'amener progressivement l'EN à s'engager dans une dynamique de modification de ses pratiques en cherchant à le convaincre grâce à des faits objectifs (Serres, \& Moussay, 2014) sans toutefois trop grandement le déstabiliser (Watson, 2006). En Phase 2, le travail de formation engagé 
par les formateurs auprès de l'EN1 est de nature différente. Ils ne cherchent plus à modifier le jugement de satisfaction de l'EN1 sur son activité passée, mais davantage à l'accompagnée vers une transformation de cette dernière. Pour ce faire, ils lui « donnent un exemple de procédure envisageable » et en justifient la faisabilité en l'ancrant dans ses propres circonstances de classe. En procédant ainsi, les formateurs ont pour intention de parvenir à convaincre l'EN1 de l'intérêt de leur proposition et, à partir de là, d'engager une modification de son activité dès sa prochaine leçon. Cette activité de formation impacte d'autant plus l'activité de l'EN qu'elle ancre les conseils dans son contexte professionnel (Coffey, 2010) et l'exemplarise par des procédures concrètes et accessibles (Schunk, 1996). Enfin en Phase 3, confrontés à un nouveau jugement d'insatisfaction de l'EN, les formateurs s'efforcent de la soutenir en trouvant un «moyen d'adapter ce qu'elle faisait pour que cela continue à être efficace» malgré les évolutions de son contexte d'enseignement. Pour ce faire, le FU1 s'engage dans une activité singulière qu'il signifie lors de son autoconfrontation en suivant la règle: [ Encourager l'EN à persévérer» vaut pour "trouver rapidement un moyen d'adapter ce qu'elle faisait » ce qui obtient comme résultats qu'elle retrouve une efficacité dans sa façon de faire et "ne baisse pas les bras»]. Ce résultat entre en écho avec ceux de la littérature qui mettent en évidence l'intérêt pour les formateurs de soutenir la persévérance des EN (Clarke, \& Jarvis-Selinger, 2005 ; Desmeules, \& Hamel, 2017).

Finalement, d'un point de vue théorique, ces résultats permettent donc de soutenir que la trajectoire de satisfaction de l'EN est directement couplée à l'efficacité de son activité professionnelle, mais aussi, dans le même temps et de façon consubstantielle, aux activités de formation engagées par les formateurs (comme cela est déjà établi pour la satisfaction professionnelle notamment par Duffy et Lent (2009). De façon complémentaire, ils permettent de mettre en évidence que ces activités de formation visent tout à la fois à fixer les critères (de satisfaction) partagés au sein de la communauté professionnelle enseignante et à aider à l'aménagement des conditions (notamment ici d'enseignement) d'atteinte des résultats attendus selon ces mêmes critères (Clot, 2008 ; 2010). À ce titre, ces résultats complètent ceux de Saussez et Yvon (2014) pour lesquels les EN seraient en difficulté sans aménagement de leur situation de travail. À cet égard, ils permettent sans doute de poser les conditions de la constitution de véritables « collectif de formation » nécessaires à l'accompagnement effectif des EN en formation (Cartaut, \& Bertone, 2009 ; Cuenca, Schmeichel, Butler, Dinkelman, \& Nichols, 2011 ; LoCasale-Crouch, Davis, Wiens, \& Pianta, 2012 ; Serres, \& Moussay, 2014).

Plus largement, les résultats détaillés et discutés montrent combien la dynamique intrinsèque du couplage [activité - satisfaction] de l'EN ne peut être appréhendée en dehors du travail de trajectoire engagé dans chacun des situations de travail et de formation, c'est-à-dire l'ensemble des activités du collectif de formateurs. À ce titre, ils permettent de soutenir que la compréhension de ce que vivent les EN au travail ne peut se faire sans la compréhension de leurs activités de travail nécessitant elles-mêmes la compréhension des activités de formation qui les ont potentiellement initiées et accompagnées.

Bien que des limites, notamment méthodologiques, puissent être avancées ${ }^{2}$ certaines conséquences technologiques en matière de formation professionnelle des EN peuvent être tracées (Bertone, \& Chaliès, 2015). Les résultats de cette étude invitent à discuter principalement deux aspects des dispositifs de formation. Tout d'abord, ils invitent à ce que les situations d'entretien soient aussi des occasions de satisfaction pour engager les 
EN dans des trajectoires de formation singulière. En complément, ils invitent à mieux accompagner les EN dans ces trajectoires en faisant des situations de travail de véritables situations de formation, par leurs aménagements singuliers (par exemple, un aménagement de la situation de travail pour favoriser le constat de résultat attendu de type co-teaching (Michel \& Bertone, 2017).

\section{BIBLIOGRAPHIE}

Amathieu, J., \& Chaliès, S. (2014). Formation, satisfaction professionnelle et santé au travail des enseignants (note de synthèse). Carrefours de l'éducation, 38, 211-238.

Amathieu, J., Escalié, G., Bertone, S. \& Chaliès, S. (2018). Formation par alternance et satisfaction professionnelle des enseignants novices. Les Sciences de l'éducation - Pour l'Ère nouvelle, 51, 87-116. https://doi.org/10.3917/lsdle.514.0087

Bertone, S., \& Chaliès, S. (2015). Construire un programme de recherche technologique sur laformation des enseignants : choix épistémologiques et théoriques. Activités [En ligne], 12-2. mis en ligne le 15 octobre 2015, consulté le 02 octobre 2016. URL : http://activites.revues.org/1088 ; https://doi.org/10.4000/activites.1088

Bertone, S., Chaliès, S., \& Clot, Y. (2009). Contribution d'une théorie de l'action à la conceptualisation et à l'évaluation des pratiques réflexives dans les dispositifs de formation initiale des enseignants. Le Travail Humain, 72(2), 105-125. https://doi.org/10.3917/th.722.0105

Billaudeau N., \& Vercambre-Jacquot M.-N. (2015). Satisfaction professionnelle des enseignants du secondaire : quelles différences entre public et privé ? Éducation \& formations, 88-89, 201-220.

Borman G.G., \& Dowling, N.M. (2008). Teacher Attrition and Retention: A Meta-Analytic and Narrative Review of the Research. Review of Educational Research, 78(3), 367-409.

Boyle, J.G., Borg, M.G., Falzon, J.M., \& Baglioni, A. (1995). A structural model of the dimensions of teacher stress. British Journal of Educational Psychology, 65, 49-67.

Canguilhem, G. (1943). Le normal et le pathologique. Paris : PUF.

Cartaut, S., \& Bertone, S. (2009). Co-analysis of work in the triadic supervision of preservice teachers based on neo-Vygotskian activity theory: Case study from a French University Institute of Teacher Training. Teaching and Teacher Education, 25(8), 1086-1094.

Cash, M. (2009). Normativity is the mother of intention: Wittgenstein, normative practices and neurological representations. New Ideas in Psychology, 27, 133-147.

Chaliès, S. (2016). Tutorat et construction des compétences professionnelles par les enseignants stagiaires. Recherche et formation, 83, 33-48.

Chaliès, S., Amathieu, J., \& Bertone, S. (2013). Former les enseignants pour accroître leur satisfaction au travail : propositions théoriques et illustrations empiriques. Le Travail Humain, 76(3), 309-334. 
Chaliès, S., \& Bertone, S. (2017). And if L. Wittgenstein helped us to think differently about teacher education? In M.A. Peters, \& J. Stickney (Eds.), A Companion to Wittgenstein on Education: Pedagogical Investigations (pp. 659-673). Springer, Editors

Chaliès, S., Bruno, F., Méard, J., \& Bertone, S. (2010). Training preservice teachers rapidly: the need to articulate the training given by university supervisors and cooperating teachers. Teaching and Teacher Education, 26, 764-774.

Chauviré C. (1992). Introduction (50 p.) à Leçons et conversations de Wittgenstein sur l'éthique, l'esthétique et la croyance religieuse. Paris : Gallimard.

Chauviré, C. (2010). Wittgenstein en héritage. Philosophie de l'esprit, épistémologie, pragmatisme. Paris : Kimé.

Clarke, A., \& Jarvis -Selinger, S. (2005). What the teaching perspectives of cooperating teachers tell us about their advisory practices. Teaching and Teacher Education, 21, 65-78.

Ciavaldini-Cartaut, S., \& Blaya, C. (2018, soumis). Climat scolaire et satisfaction professionnelle des enseignants : un enjeu pour la qualité « du vivre et travailler ensemble » au sein d'établissements de l'éducation prioritaire. Revue Suisse des Sciences de l'Éducation.

Clot, Y. (2008). Travail et pouvoir d'agir. Paris : PUF.

Clot, Y. (2010). Le travail à cœur, pour en finir avec les risques psychosociaux. Paris : La Découverte.

Clot, Y., \& Lhuilier D. (2015). Perspectives en clinique du travail. Toulouse : Erès. https://doi.org/ 10.3917/eres.clot.2015.01

Coffey, H. (2010). "They taught me": the benefits of early community-based field experiences in teacher education. Teaching and Teacher Education, 26(2), 335-342.

Coutrot, T. (2018). Libérer le travail. Pourquoi la gauche s'en moque et pourquoi ça doit changer? Paris : Seuil.

Cuenca, A., Schmeichel, M., Butler, B., Dinkelman, T., \& Nichols, J. (2011). Creating a "third space" in student teaching: Implications for the university supervisor's status as outsider. Teaching and Teacher Education, 27, 1068-1077.

Davis, A. (2009). Examples as method? My attempts to understand assessment and fairness in the spirit of the later Wittgenstein. Journal of Philosophy of Education, 43, 371-389.

Dejours, C (2016). Souffrance et plaisir au travail. L'approche par la psychopathologie du travail. Travailler, 35(1), 17-30.

Descombes, V. (2004). Le complément de sujet : enquête sur le fait d'agir de soi-même. Paris : Gallimard.

Desmeules, A., Hamel, C. (2017). Les motifs évoqués par les enseignants débutants pour expliquer leur envie de quitter le métier et les implications pour soutenir leur persévérance. Formation et profession, 25(3), 19-35. http://dx.doi.org/10.18162/fp.2017.427

Donahue, D.M. (1999). Service-learning for preservice teachers: Ethical dilemmas for practice. Teaching and Teacher Education, 15, 685-695.

Duffy, R. D., \& Lent, R. W. (2009). Test of a social cognitive model of work satisfaction in teachers. Journal of Vocational Behavior, 75, 212-223.

Fillietaz, L. (2009). La linguistique appliquée face aux réalités de la formation professionnelle. Bulletin suisse de linguistique appliquée, 90, 1-15. 
Fotinos, G., Horenstein, J. M. (2011). La qualité de vie au travail dans les lycées et collèges : le burnout des enseignants. Paris, Collection MGEN Eds.

Gaziel, H., \& Wasserstein-Warnet, M. (2005). Les facteurs influençant la satisfaction du travail des enseignants dans des contextes organisationnels et socio-culturels différents. Les Sciences de l'éducation - Pour l'Ère nouvelle, 38(4), 111-131.

Gold, Y. (1996). Beginning teacher support : Attrition, mentoring, and induction. In J. Sikula, T. J. Buttery, \& E. Guyton (Eds.), Handbook of research on teacher education (p. 548-594). New York, NY : Macmillan.

Goldring, R., Taie, S., \& Riddles, M. (2014). Teacher Attrition and Mobility: Results from the 2012-13 Teacher Follow-Up Survey. First Look. NCES 2014-077

Jones Johnson, G., \& Johnson, W.R. (2000). Perceived over qualification and dimensions of job satisfaction: A longitudinal analysis. Journal of Psychology, 34(5), 537-556.

Lachmann, H., Larose, C., Penicaud, C., \& Moleux, M. (2010). Bien-être et efficacité au travail. 10 propositions pour améliorer la santé psychologique au travail. Rapport fait à la demande du Premier ministre, février.

Lähteenmäki, M. (2003). On rules and rule following: obeying rules blindly. Language and Communication, 23(1), 45-61.

Lanouzière, H. (2019). Mal-être ou satisfaction au travail dans un monde en mutation. Pourquoi et comment travailler autrement ? Regards, 1(1), 65-74. https://doi.org/10.3917/regar.055.0065

Lantheaume, F., \& Helou, C. (2008). Les difficultés au travail des enseignants. Exception ou part constitutive du métier? Recherche et Formation, 57, 65-78.

Lave, J., \& Wenger, E. (1991). Situated learning: Legitimate peripheral participation. Cambridge, UK : Cambridge University Press.

Le Blanc, G. (2008). Canguilhem et les normes. Paris, France : Presses Universitaires de France. https://doi.org/10.3917/puf.lebla.2010.01

Lee, M.S.M., Lee, M.B., Liao, S.C., \& Chang, F.T. (2009). Relationship Between Mental Health and Job Satisfaction Among Employees in a Medical Department of Laboratory Medicine. Journal of the Formosan Medical Association, 108(2). 146-154.

Legrand, D. (2005). Pre-reflective self-as-subject from experiential and empirical perspectives. Consciousness and Cognition, 16, 583-599.

Lent, R.W., \& Brown, S.D. (2008). Social cognitive career theory and subjective well-being in the context of work. Journal of Career Assessment, 16, 6-21.

Lhuilier, D., \& Litim, M. (2009). Le rapport santé-travail en psychologie du travail. Mouvements, 58(2), 85-96. https://doi.org/10.3917/mouv.058.0085

Livet, P. (1993). Théorie de l'action et conventions. In P. Ladrière, P. Pharo, \& L. Quéré (Eds.), La théorie de l'action. Le sujet pratique en débat (pp. 291-319). Paris : CNRS Éditions.

LoCasale-Crouch, J., Davis, E., Wiens, P., \& Pianta, R. (2012). The role of the mentor in supporting new teachers: associations with self-efficacy, reflection, and quality. Mentoring and Tutoring: Partnership in Learning, 20(3), 303-323. http://dx.doi.org/10.1080/13611267.2012.701959

Locke, E. (1976). The nature and causes of job satisfaction. In M. Dunette (Ed.), Handbook of industrial and organizational psychology. Chicago : RandMcNally. 
Maroy, C. (2008). Perte d'attractivité du métier et malaise enseignant. Le cas de la Belgique. Recherches et formation, 57, 23-38.

McInerney, D.M., Korpershoek, H., Wang, H., \& Morin, A.J.S. (2018). Teachers' occupational attributes and their psychological wellbeing, job satisfaction, occupational self and quitting intentions. Teaching and Teacher Education, 71, 145-158.

Michel, L, \& Bertone, S. (2017). Effets d'un dispositif de type coteaching sur le développement professionnel des enseignants novices : études de cas en formation universitaire. Recherches en Éducation, 30, 8-20.

Nasse, P., \& Légeron, P. (2008). Rapport sur la détermination, la mesure et le suivi des risques psychosociaux au travail. Paris : La Documentation française.

Nir A.-E., \& Bogler R. (2008). The antecedents of teacher satisfaction with professional development programs. Teaching and Teacher Education, 24, 377-386.

Ogien, A. (2007). Les formes sociales de la pensée. La sociologie après Wittgenstein. Paris : Armand Colin. Olszewska, B., \& Quéré, L. (2009). Erreurs pratiques, fautes et incongruités. In C. Chauviré, Ogien, A., \& Quéré, L. (Eds), Dynamiques de l'erreur (pp. 167-205). Paris : Éditions de l'EHESS, Raisons Pratiques, 19.

Rogoff, B., Matusov, E., \& White, C. (1996). Models of teaching and learning: participation in a community of learners. In D.R. Olson \& N. Torrance (Eds.), The handbook of education and human development: New models of learning, teaching and schooling (pp. 388-414). Oxford : Basil Blackwell Publishers.

Saussez, F., \& Yvon, F. (2014) Problématiser l'usage de la co-analyse de l'activité en formation initiale à l'enseignement. In L. Paquay, P. Perrenoud, M. Altet, J. Desjardins \& R. Etienne (Eds.), Travail réel des enseignants et formation. Quelle référence au travail des enseignants dans les objectifs, les dispositifs et les pratiques? (pp. 113-126). Bruxelles : De Boeck.

Schunk, D. H. (1996). Goal and self-evaluative influences during children's cognitive skill Practical Assessment, Research \& Evaluation, 11(10), 359-382.

Schwarz, N. (2007). Attitude construction : Evaluation in context. Social Cognition, 25(5), 638-656.

Serres, G., \& Moussay, S. (2014). Activités des formateurs d'enseignants : quelles fonctions pour quels objectifs ? État de l'art. Conférence de consensus-dissensus, chaire de l'UNESCO, Lyon, France.

Skaalvik, E.M., \& Skaalvik, S. (2015). Job Satisfaction, Stress and Coping Strategies in the Teaching Profession-What Do Teachers Say? International Education Studies, 8(3), 181-192.

Skaalvik, E.M., \& Skaalvik, S. (2017). Motivated for teaching? Associations with school goal structure, teacher self-efficacy, job satisfaction as emotional exhaustion. Teaching and Teacher Education, 67, 152-160.

Strauss, A. (1992). La trame de la négociation, Textes réunis et présentés par Isabelle Baszanger. Paris : L'Harmattan.

Strauss, A.L, Fagerhaugh, S., Suczek, B., \& Wiener, C. (1985). The social organization of medical work. Chicago, IL : University of Chicago Press.

Taylor, C. (1997). La liberté des modernes. Paris : PUF.

Travis, C. (2003). Les liaisons ordinaires. Paris : Vrin. 
Von der Embse, N.P., Sandilos, L.E., Pendergast, L., \& Mankin, A. (2016). Teacher stress, teachingefficacy, and job satisfaction in response to test-based educational accountability policies, Learning and Individual Differences, 50, 308-317.

Watson, S. (2006). Novice Science Teachers: Expectations and Experiences. Faculty Publications and Presentations, 16, 279-290. http://digitalcommons.liberty.edu/educ_fac_pubs/16

Williams, M. (2002). Tout est-il interprétation? In C. Chauviré, \& A. Ogien (Eds.), La régularité (pp. 207-233). Paris : EHESS.

Winch, P. (2009). L'idée d'une science sociale et sa relation à la philosophie. Paris : Gallimard.

Wittgenstein, L. (1992). Les cours de Cambridge 1932-1935. Mauvezin : TER.

Wittgenstein, L. (1996). Remarques philosophiques. Paris : Gallimard.

Wittgenstein, L. (2004). Recherches philosophiques. Paris : Gallimard.

Yennek, N. (2015). La satisfaction en formation d'adultes. Savoirs, 38(2), 9-54.

\section{NOTES}

1. Ce dispositif concerne deux EN, suivies et consiste en un accompagnement suivi de deux EN en formation en alternance entre leur établissement scolaire et le centre de formation (Institut National Supérieur du professorat et de l'éducation (INSPE)

2. Le travail entamé et exposé ici a été poursuivi pour identifier le jugement de satisfaction par une analyse du langage (langage du corps, langage verbal...)

\section{RÉSUMÉS}

Différents rapports institutionnels (Lachman, Nasse-Légeron) traitent de la question du bien-être des travailleurs. En leur sein, une relation est régulièrement établie entre la santé des enseignants et la nature des activités qu'ils mènent au sein des établissements scolaires. Du point de vue des théories de l'activité, ce consensus amène à une principale réserve. En assimilant la satisfaction professionnelle à un état psychologique stable et " a situé », ces études la désolidarise de l'activité effective des enseignants, activité nécessairement située en contexte de classe et parlà même constamment soumise à des circonstances situationnelles sans cesse renouvelées. En prenant appui sur quatre postulats empruntés à un programme de recherche mené en anthropologie culturaliste (Auteurs, 2017), cette étude a pour ambition d'aider au dépassement de cette réserve. Pour ce faire, elle propose successivement (i) une conceptualisation théorique originale permettant d'appréhender selon un point de vue qualitatif le couplage activité satisfaction professionnelle, (ii) une démarche méthodologique permettant de rendre compte du caractère situé et dynamique de ce couplage et, enfin, (iii) une illustration et une discussion du caractère heuristique de ces propositions théoriques et méthodologiques à partir de la présentation de résultats empiriques extraits d'une étude de cas.

As in other professional fields, there would seem to be a consensus regarding links that can be established between teachers' health and the nature of their activities in the workplace. From the 
activity theory standpoint, this consensus leads to one main restriction. By equating job satisfaction with a stable and "situated" psychological state, these studies dissociate it, so to speak, from the actual activity of teaching one that is necessarily situated in a class context and thus constantly subject to circumstances and changing situations. Based on these postulates, borrowed from a research program conducted in cultural anthropology (Auteurs, 2017), this study aims to help overcome this issue. To this end, we successively propose (i) an original theoretical conceptualization that makes it possible to apprehend, from a qualitative point of view, the coupling between activity and professional satisfaction, (ii) a methodological approach to account for the location and dynamic nature of this coupling and (iii) an illustration and discussion of the heuristic nature of these theoretical and methodological propositions based on the presentation of empirical results extracted from a case study.

\section{INDEX}

Mots-clés : activités, satisfaction professionnelle, trajectoire de satisfaction, formation des enseignants, anthropologie culturaliste

Keywords : activities, job satisfaction, satisfaction trajectory, teacher training, culturalist anthropology

\section{AUTEURS}

\section{JÉRÔME AMATHIEU}

INSPE de Toulouse Midi-Pyrénées - Université de Toulouse - UMR «Éducation Formation Travail Savoirs »- jerome.amathieu@univ-tlse2.fr

\section{SÉBASTIEN CHALIÈS}

INSPE de Toulouse Midi-Pyrénées - Université de Toulouse - UMR « Éducation Formation Travail Savoirs »- sebastien.chalies@univ-tlse2.fr 